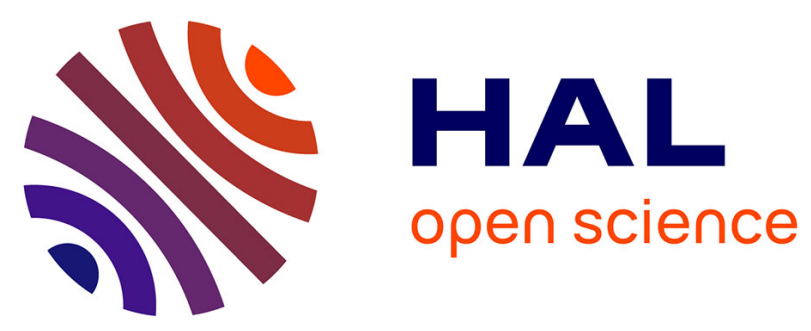

\title{
An intestinal zinc sensor regulates food intake and developmental growth
}

Siamak Redhai, Clare Pilgrim, Pedro Gaspar, Lena Van Giesen, Tatiana

Lopes, Olena Riabinina, Théodore Grenier, Alexandra Milona, Bhavna

Chanana, Jacob Swadling, et al.

\section{To cite this version:}

Siamak Redhai, Clare Pilgrim, Pedro Gaspar, Lena Van Giesen, Tatiana Lopes, et al.. An intestinal zinc sensor regulates food intake and developmental growth. Nature, 2020, 580 (7802), pp.263-268. 10.1038/s41586-020-2111-5 . hal-02998956

\section{HAL Id: hal-02998956 https://hal.science/hal-02998956}

Submitted on 12 Nov 2020

HAL is a multi-disciplinary open access archive for the deposit and dissemination of scientific research documents, whether they are published or not. The documents may come from teaching and research institutions in France or abroad, or from public or private research centers.
L'archive ouverte pluridisciplinaire HAL, est destinée au dépôt et à la diffusion de documents scientifiques de niveau recherche, publiés ou non, émanant des établissements d'enseignement et de recherche français ou étrangers, des laboratoires publics ou privés. 


\section{An intestinal zinc sensor regulates food intake and developmental growth}

https://doi.org/10.1038/s41586-020-2111-5

Received: 17 May 2019

Accepted: 18 February 2020

Published online: 18 March 2020

Check for updates

\author{
Siamak Redhai ${ }^{1,2,10}$, Clare Pilgrim $^{1,2,10}$, Pedro Gaspar ${ }^{1,2}$, Lena van Giesen ${ }^{3}$, Tatiana Lopes ${ }^{1,2}$, \\ Olena Riabinina ${ }^{1,2,9}$, Théodore Grenier ${ }^{4}$, Alexandra Milona', Bhavna Chanana ${ }^{1,2}$, \\ Jacob B. Swadling ${ }^{1,2}$, Yi-Fang Wang ${ }^{1}$, Farah Dahalan ${ }^{5,6}$, Michaela Yuan ${ }^{7}$, \\ Michaela Wilsch-Brauninger ${ }^{7}$, Wei-hsiang Lin ${ }^{8}$, Nathan Dennison ${ }^{5}$, Paolo Capriotti ${ }^{5}$, \\ Mara K. N. Lawniczak ${ }^{6}$, Richard A. Baines ${ }^{8}$, Tobias Warnecke ${ }^{1,2}$, Nikolai Windbichler ${ }^{5}$, \\ Francois Leulier ${ }^{4}$, Nicholas W. Bellono ${ }^{3}$ \& Irene Miguel-Aliaga, ${ }^{1,2 \bowtie}$
}

\begin{abstract}
In cells, organs and whole organisms, nutrient sensing is key to maintaining homeostasis and adapting to a fluctuating environment ${ }^{1}$. In many animals, nutrient sensors are found within the enteroendocrine cells of the digestive system; however, less is known about nutrient sensing in their cellular siblings, the absorptive enterocytes ${ }^{1}$. Here we use a genetic screen in Drosophila melanogaster to identify Hodor, an ionotropic receptor in enterocytes that sustains larval development, particularly in nutrient-scarce conditions. Experiments in Xenopus oocytes and flies indicate that Hodor is a $\mathrm{pH}$-sensitive, zinc-gated chloride channel that mediates a previously unrecognized dietary preference for zinc. Hodor controls systemic growth from a subset of enterocytes-interstitial cells-by promoting food intake and insulin/ IGF signalling. Although Hodor sustains gut luminal acidity and restrains microbial loads, its effect on systemic growth results from the modulation of Tor signalling and lysosomal homeostasis within interstitial cells. Hodor-like genes are insect-specific, and may represent targets for the control of disease vectors. Indeed, CRISPR-Cas9 genome editing revealed that the single hodor orthologue in Anopheles gambiae is an essential gene. Our findings highlight the need to consider the instructive contributions of metals-and, more generally, micronutrients-to energy homeostasis.
\end{abstract}

To investigate nutrient sensing in enterocytes, we selected 111 putative nutrient sensors in D. melanogaster on the basis of their intestinal expression and their predicted structure or function (Extended Data Fig. 1a, Supplementary Information). Using two enterocyte-specific driver lines, we downregulated their expression in midgut enterocytes throughout development under two dietary conditions, nutrient-rich and nutrient-poor; we reasoned that dysregulation of nutrient-sensing mechanisms may increase or reduce the normal period of larval growth, and might do so in a diet-dependent manner (Extended Data Fig. 1b-d). Enterocyte-specific knockdown of the gene CG1134O, also referred to as $\mathrm{pHCl}-2^{2}$, resulted in developmental delay. This delay was exacerbated, and was accompanied by significantly reduced larval viability, under nutrient-poor conditions (Fig. 1a, Extended Data Figs. 1h, 2b); these phenotypes were confirmed using a second $R N A i$ transgene and a new CG1134O mutant (Fig. 1b, c, Extended Data Fig. 1e-i). In the tradition of naming Drosophila genes according to their loss-of-function phenotype, we have named CG11340 'hodor'-an acronym for 'hold on, don't rush', in reference to the developmental delay.
A transcriptional reporter revealed that Hodor was expressed in the intestine ${ }^{3}$. A new antibody (Extended Data Fig. 2a, b) revealed that Hodor expression was confined to enterocytes in two midgut portions that are known to store metals: the copper cell region and the iron cell region (Fig. $1 \mathrm{~d}-\mathrm{h}$ ). Within the copper cell region, Hodor was expressed only in so-called interstitial cells (Fig. 1e,f,g). hodor-Gal4 was also present in the interstitial cells of the copper cell region; however, in our experimental conditions and in contrast to published results, it was not detected in the iron cell region ${ }^{3}$ (Fig. 1e, Extended Data Fig. 2d). Apart from the intestine, Hodor was found only in principal cells of the excretory Malpighian tubules ${ }^{2,3}$ (Fig. 1d, e). To identify the cells from which Hodor controls systemic growth, we conducted region- or celltype-specific downregulation and rescue experiments (Extended Data Figs. 1b, 2d-g). Only fly lines in which hodor was downregulated in interstitial cells showed slowed larval development (Fig. 1a, i-k, Extended Data Figs. 1j, 2c-h). This developmental delay persisted when hodor knockdown was induced post-embryonically during larval growth (Fig. 1l), and was rescued only in fly lines in which hodor expression was

${ }^{1}$ MRC London Institute of Medical Sciences, London, UK. ${ }^{2}$ Institute of Clinical Sciences, Faculty of Medicine, Imperial College London, London, UK. ${ }^{3}$ Department of Molecular and Cellular Biology, Harvard University, Cambridge, MA, USA. ${ }^{4}$ Institut de Génomique Fonctionnelle de Lyon (IGFL), Université de Lyon, ENS de Lyon, CNRS UMR 5242 , Lyon, France. ${ }^{5}$ Department of Life Sciences, Imperial College London, London, UK. ${ }^{6}$ Malaria Programme, Wellcome Sanger Institute, Cambridge, UK. ${ }^{7}$ Max Planck Institute of Molecular Cell Biology and Genetics, Dresden, Germany. ${ }^{8}$ Division of Neuroscience and Experimental Psychology, School of Biological Sciences, Faculty of Biology, Medicine and Health, University of Manchester, Manchester Academic Health Science Centre, Manchester, UK. ${ }^{9}$ Present address: Department of Biosciences, Durham University, Durham, UK. ${ }^{10}$ These authors contributed equally: Siamak Redhai, Clare Pilgrim.

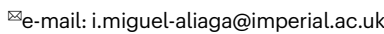




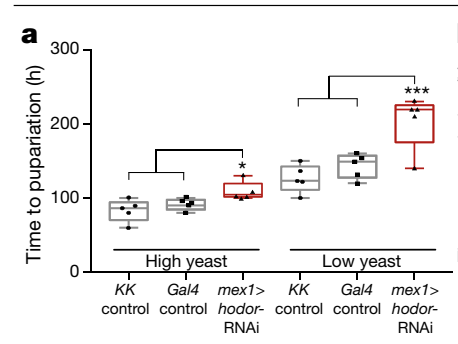

d
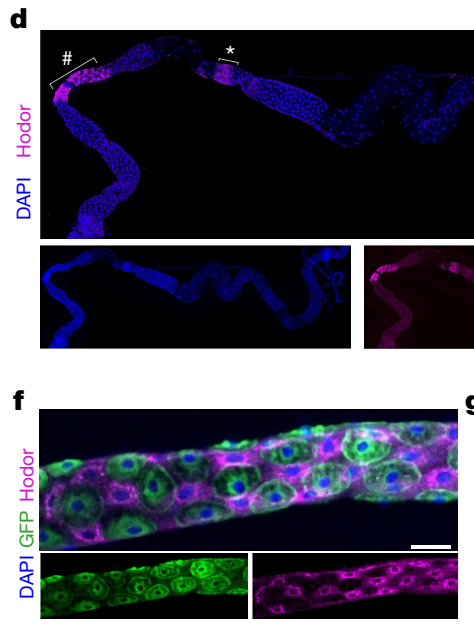

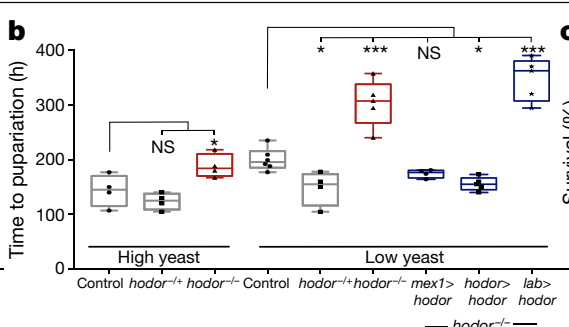

- hodor-1-

e

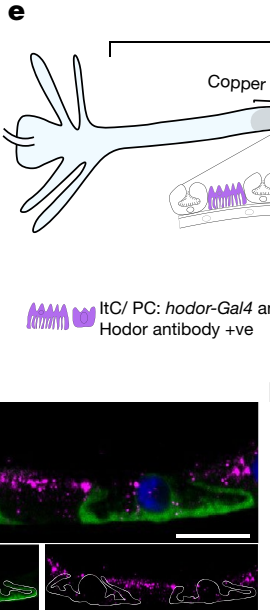

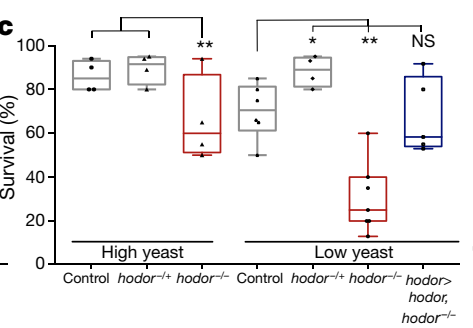

Midgut

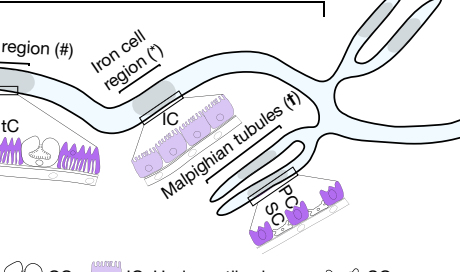

ItC

(8) CC Lator Hodor antibody +ve

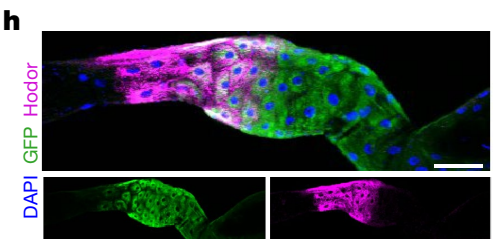

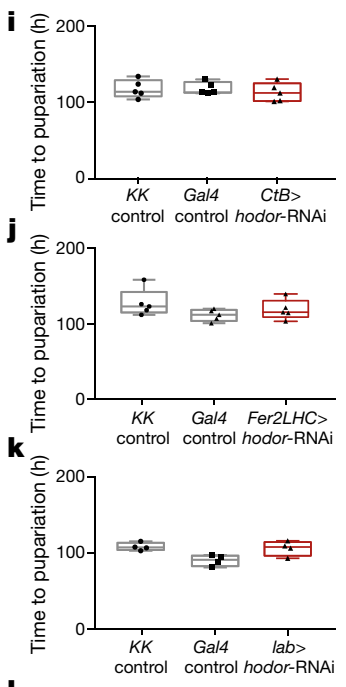

'

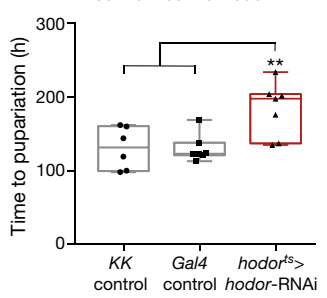

Fig. 1 Intestinal Hodor sustains larval growth. a, Enterocyte-specific (mex1Gal4-driven) hodor knockdown increases time to pupariation, particularly in nutrient-poor (low-yeast) conditions. b, Developmental delay of hodor mutants (increased time to pupariation) in both nutrient-rich (high-yeast) and nutrientpoor (low-yeast) conditions. The delay can be fully rescued by overexpressing hodor in interstitial cells and Malpighian tubule principal cells (using a hodorGal4 driver), in migdut enterocytes (using a mex1-Gal4 driver), but not in copper cells (using a labial (lab)-Gal4 driver).c, The nutrient-dependent reduced viability of hodor mutants is rescued by hodor-Gal4-driven hodor reexpression. d, Hodor expression in the copper cell region (\#), the iron cell region $(*)$ and the Malpighian tubules $(\dagger)$ of a third-instar larval midgut. Expression in the large flat cell region flanked by the copper and iron cell regions was observed only sporadically. e, Schematic of Hodor expression. CC copper cells; IC, iron cells; ItC, interstitial cells; $\mathrm{PC}$, principal cells; SC, stellate cells.f, Hodor-positive interstitial cells are interspersed among copper cells (GFP-positive (lab>mCD8-GFP), Hodor-negative). g, Hodor is found on the apical (luminal, up) side of interstitial cells, flanked by GFP-expressing copper

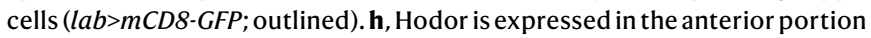
of the iron cell region (Fer1HCH-GFP-positive). $\mathbf{i}-\mathbf{k}$, Knockdown of hodor in principal cells (using CtB-Gal4) (i), iron cells (using Fer2LCH-Gal4) (j), or copper cells (using lab-Gal4) (k) all fail to alter larval development. I, Post-embryonic hodor knockdown in interstitial and Malpighian tubule principal cells (by means of hodor-Gal4, tub-Ga8O $0^{t s}$ (denoted hodor ${ }^{\text {ts }}$ )-driven hodor RNAi) increases the time to pupariation. See Supplementary Information for sample sizes and full genotypes. One-way analysis of variance (ANOVA) with Tukey post hoc tests were used for all graphs. ${ }^{*} P<0.05,{ }^{* *} P<0.01,{ }^{* * *} P<0.001$. Box plots: line, median; box, 75th-25th percentiles; whiskers, minimum to maximum. Scale bars, $1 \mathrm{~mm}$ (d); $40 \mu \mathrm{m}$ (f); $20 \mu \mathrm{m}$ (g); $100 \mu \mathrm{m}$ (h). re-instated in cell types that included interstitial cells (Fig. 1b, c). The fat body (analogous to liver and adipose tissue) has long been known to couple nutrient availability with developmental rate ${ }^{4,5}$; however, recent studies have revealed contributions from the intestine, particularly in nutrient-poor conditions ${ }^{6,7}$. Our findings confirm a role for the intestine in coupling nutrient availability with larval growth, and further implicate a subpopulation of enterocytes-interstitial cells-as important mediators. Interstitial cells were described decades ago in blowfly ${ }^{8}$, but had remained relatively uncharacterized since; their name refers only to their position ${ }^{9}$, interspersed among the acid-secreting copper cells that control microbiota loads ${ }^{10-13}$.

\section{Hodor control of systemic growth}

We established that the lethality of hodor mutation or knockdown was apparent only during the larval period (Extended Data Fig. 3a). The development of hodor mutants was slower throughout larval life, and surviving mutants attained normal pupal and adult sizes (Extended Data Fig. 3b-d). Consistent with previous findings ${ }^{12}$, hodor mutation or knockdown was found to reduce luminal acidity in the copper cell region (Extended Data Fig. 4a, b), suggesting a role specifically for interstitial cells in this process. hodor mutants also had increased gut bacterial titres, which is consistent with the observed functional defects in the copper cell region ${ }^{13}$ (Extended Data Fig. 5a). Enlarged volumes of both the lumen of the copper cell region and the interstitial cells were also apparent after 1-3 days of (delayed) larval development (Extended Data Fig. 4e); ultrastructurally, this was apparent in interstitial cells as a reduction in the complexity of their characteristic basal infoldings ${ }^{14}$ (Extended Data Fig. 4d). We were, however, able to rule out all of these defects as reasons for the developmental delay (Extended Data Figs. 4a-c, f-i, 5b, c, Supplementary Information).

During the course of these experiments, we observed that hodor mutant larvae were more translucent than control larvae (Fig. 2a). This was suggestive of peripheral lipid depletion, which we confirmed by quantifying and staining for triacylglycerides (Fig. 2b, d, e). Reduced lipid stores did not result from disrupted enterocyte integrity: the intestinal barrier of mutants was intact, both anatomically and functionally (Extended Data Fig. 3g, h). We observed that hodor mutants had less food in their intestines (Fig. 2f) and accumulated insulin-like peptide Ilp2 in their brains (nutrient-dependent Ilp2 secretion promotes larval development; its accumulation in the brain is commonly interpreted as peptide retention in the absence of transcriptional changes ${ }^{5,15}$ ) (Fig. $2 \mathrm{k}$, I). Consistent with reduced systemic insulin signalling, hodor mutant larval extracts had reduced levels of phospho-Akt and phospho-S6 kinase (Fig. 2o, Extended Data Fig. 3e). As these are all indicators of starvation, we quantified food intake and observed that it was reduced in both hodor mutant larvae and in hodor knockdowns targeting interstitial cells (Fig. 2f, g, i, Extended Data Figs. 2c, 3f). Reduced food intake 

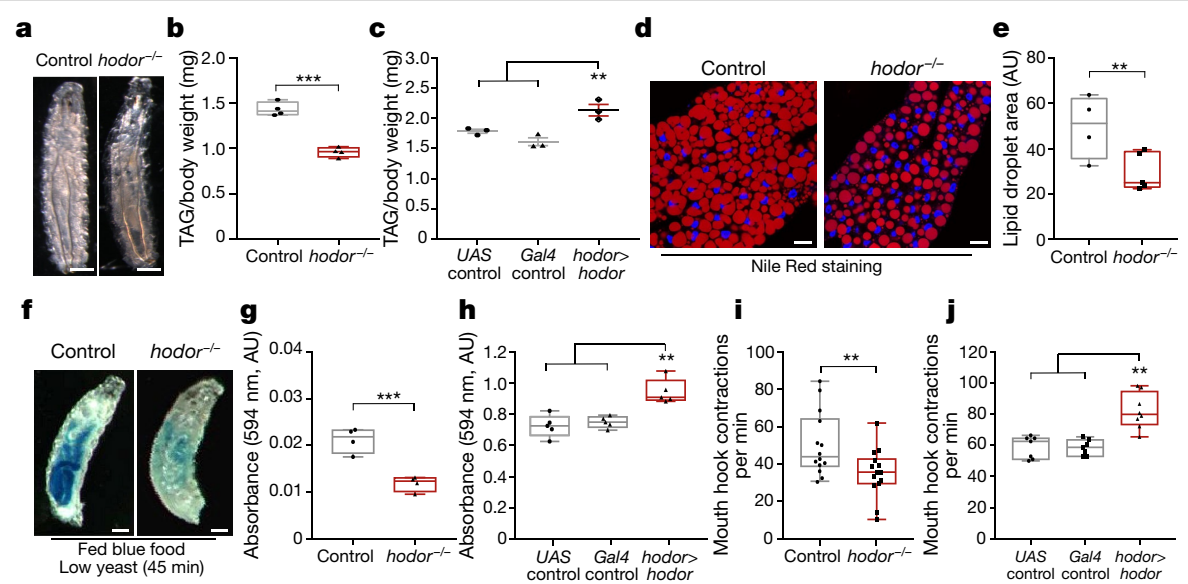

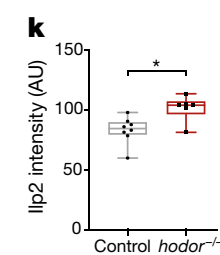

I
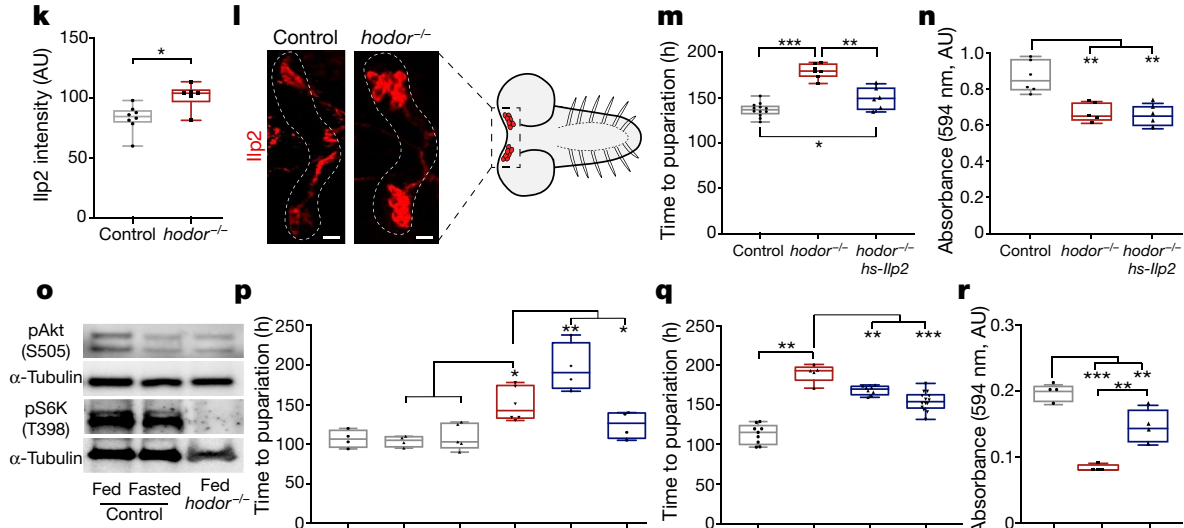

$\mathbf{p}$
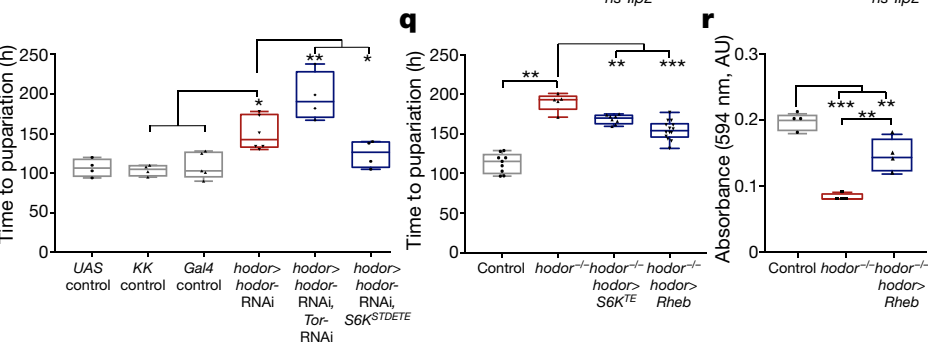

Fig. 2 | Intestinal Hodor/Tor signalling promotes food intake. a, hodor mutants are more translucent than controls. b, c, Total triacylglycerides (TAG) normalized to weight in hodor mutants (b) and larvae overexpressing hodor (c). d, Lipid droplets within the fat body of hodor mutants and controls. e, The size of fat body lipid droplets is reduced in hodor mutants. In a-e, L2 larvae were used.f, Reduced intestinal contents were found in L1 hodor mutants that were fed dye-laced food for $45 \mathrm{~min} . \mathbf{g}-\mathbf{j}$, Food intake $(\mathbf{g}, \mathbf{h})$ or mouth hook contraction $(\mathbf{i}, \mathbf{j})$ quantifications for L1 hodor mutants $(\mathbf{g}$, i) or L2 larvae overexpressing hodor in hodor-expressing cells $(\mathbf{h}, \mathbf{j}) . \mathbf{k}, \mathbf{l}$, Ilp2 staining (quantification, $\mathbf{k}$ and representative images, $\mathbf{l}$ ) of $\mathrm{L} 2$ brains of controls compared with hodor mutants. m, n, Ectopic Ilp2 expression ( $h s$-Ilp2) rescues the developmental delay of hodor mutants (m), but not their food intake (n). o, Reduced pAkt and pS6K in L2 hodor mutants compared to controls. p, The developmental delay of hodor knockdowns is exacerbated or rescued when the Tor pathway is

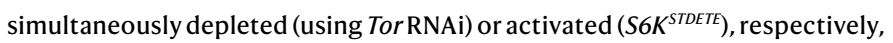
specifically in hodor-expressing cells. These manipulations did not affect the development of wild-type larvae (Extended Data Fig. 3j). q, The developmental delay of hodor mutants is rescued by activation of the Tor pathway (using $S 6 K^{T E}$, which is weaker than $S 6 K^{\text {STDETE}}$, or UAS-Rheb) specifically in hodor-expressing cells. r, The reduced food intake of L2 hodor mutants is rescued by Tor pathway activation specifically in hodor-expressing cells (hodor $>$ Rheb). See Supplementary Information for sample sizes and full genotypes. MannWhitney $U$-tests or ordinary one-way ANOVA with Tukey post hoc tests were used for two-group comparisons, or for comparisons involving more than two groups, respectively. ${ }^{*} P<0.05,{ }^{* *} P<0.01,{ }^{* * *} P<0.001$. Box plots: line, median; box, 75th-25th percentiles; whiskers, minimum to maximum. Scale bars, $500 \mu \mathrm{m}$ (a); $20 \mu \mathrm{m}$ (d); $100 \mu \mathrm{m}$ (f); $15 \mu \mathrm{m}$ (I). was apparent soon after hatching and persisted throughout larval development (Fig. 2f, g, Extended Data Fig. 3f). Ectopic expression of Ilp2-which rescues developmental delay in larvae that lack insulin-like peptides $^{15}$-in hodor mutants partially rescued their developmental delay, but did not increase their food intake (Fig. 2m, n). An 'instructive' link between intestinal Hodor and food intake was further suggested by the overexpression of hodor in otherwise wild-type enterocytes; this resulted in larvae that ate more and developed at a normal rate, but had increased lipid stores (Fig. 2c, h, j, Extended Data Fig. 3i). Therefore, Hodor controls larval growth from a subset of enterocytes by promoting food intake and systemic insulin signalling. In its absence, larvae fail to eat sufficiently to proceed through development at the normal rate and are leaner. When present in excess, Hodor causes larvae to eat more and accumulate the energy surplus as fat.

In fly adipose tissue, amino acid availability activates Tor signalling to promote systemic growth ${ }^{4}$. We therefore combined hodor knockout or knockdown with genetic manipulations to alter Tor signalling. In flies with reduced or absent Hodor function, decreasing or increasing Tor signalling in hodor-expressing cells exacerbated or rescued the developmental delay, respectively (Fig. 2p, q, Extended Data Fig. 3j). The reduced food intake of hodor mutants was also significantly rescued by activation of Tor signalling in hodor-expressing cells (Fig. 2r, Extended Data Fig. 3j, k). Genetic targeting of Rag GTPases or the Gator1 complex in these cells failed to affect the developmental delay of hodor mutants (Extended Data Fig. 3l), which could suggest non-canonical regulation of Tor signalling in Hodor-expressing cells. The systemic effects of Hodor on food intake and larval growth are therefore modulated by Tor signalling within Hodor-expressing interstitial cells.

\section{Hodor is a zinc-gated chloride channel}

Hodor belongs to the (typically neuronal) Cys-loop subfamily of ligandgated ion channels, and is predicted to be a neurotransmitter-gated anion channel ${ }^{16}$ (Fig. 3a, Supplementary Information). It is known to 


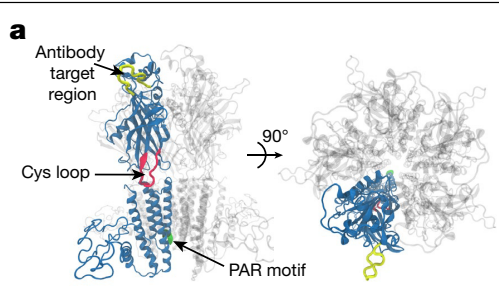

C
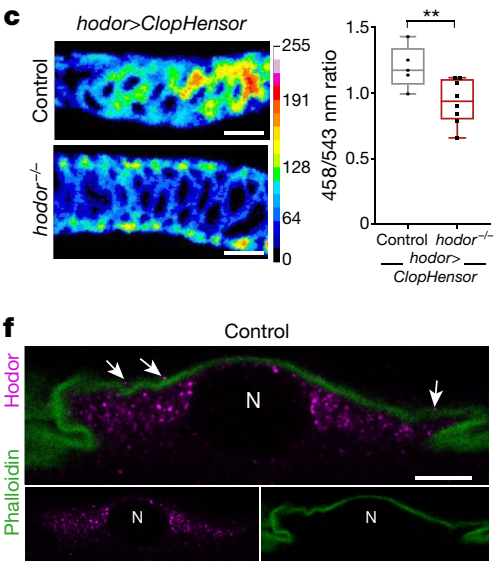

i
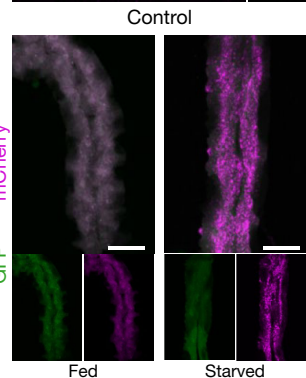

hodo

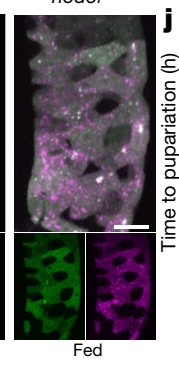

Fig. 3 | Hodor is a zinc-gated chloride channel that controls dietary zinc preference and lysosomal functions. a, Predicted structure of the pentameric Hodor complex; one monomer is shown in blue.b, Left, only oocytes that are injected with Hodor respond to zinc; middle, current-voltage $(I-V)$ curves showing zinc-activated currents; right, normalized response of the channel to zinc (estimated half-maximal effective concentration $\left(\mathrm{EC}_{50}\right): 75.20 \mu \mathrm{M}, 95 \%$ confidence interval 58.63-94.65 $\mu \mathrm{M}$ ). c, Increased intracellular chloride levels (as indicated by the decreased fluorescence intensity ratio at $458 \mathrm{~nm} / 543 \mathrm{~nm}$ of ClopHensor) in interstitial cells of L1 hodor mutants ( $20 \mathrm{mM}$ in controls, $64 \mathrm{mM}$ in hodor mutants; calibration in Extended Data Fig. 6h). Representative images $(458 \mathrm{~nm})$ are shown. d, Supplementation of a low-yeast diet with zinc increases food intake in controls, but not in hodor mutants. e, Controls-but not hodor mutants-develop a preference (positive values) for a zinc-supplemented lowyeast diet, which is significant after $45 \mathrm{~h} . \mathrm{ZnCl}_{2}$ was used in these experiments; $\mathrm{ZnSO}_{4}$ also elicited a preference, which is not shown here.f, Hodor is enriched on the apical (luminal) side of interstitial cells: on the brush border (arrows, phalloidin-positive) and intracellularly. $\mathbf{g}, \mathbf{h}$, A subpopulation of compartments

show activity in response to alkaline conditions in Xenopus oocytes ${ }^{2}$, but the acidic $\mathrm{pH}$ of the copper cell region prompted us to search for additional ligands. Although we confirmed alkaline $\mathrm{pH}$-induced Hodor activity in oocyte expression systems, Hodor did not respond to typical Cys-loop receptor ligands such as neurotransmitters or amino acids (Extended Data Table 1). Instead, our screen identified zinc as an unanticipated ligand, which elicited a strong dose-dependent response only in Hodor-expressing oocytes (Fig. 3b, Extended Data Fig. 6e); this response to zinc showed peak current amplitude values much greater than those observed in response to $\mathrm{pH}$ or to other metals such as iron or copper (Extended Data Table1). Force-field-based structural stability and binding affinity calculations (Supplementary Information) identified the amino acid pair E255 and E296 as a potential binding site for the divalent zinc ion. Mutating these residues did not abrogate the
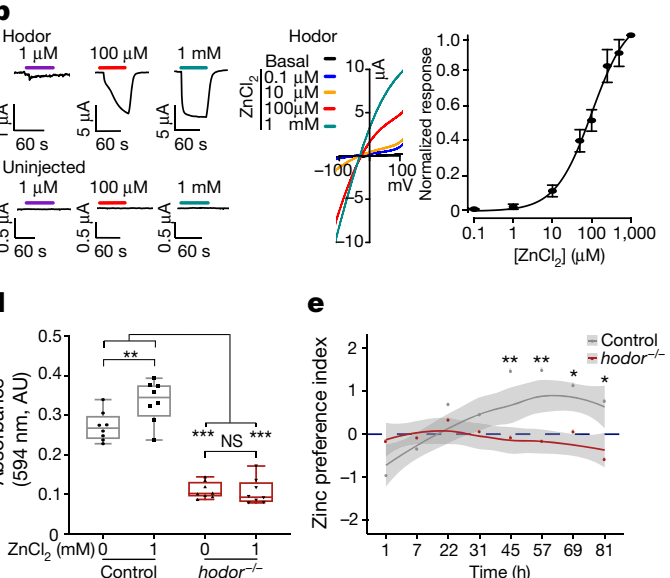

e

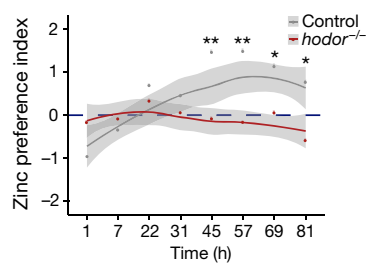

h

Control
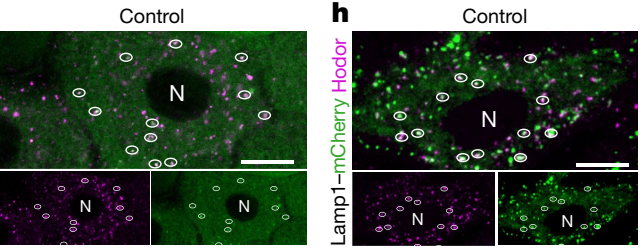

$\mathbf{k}$
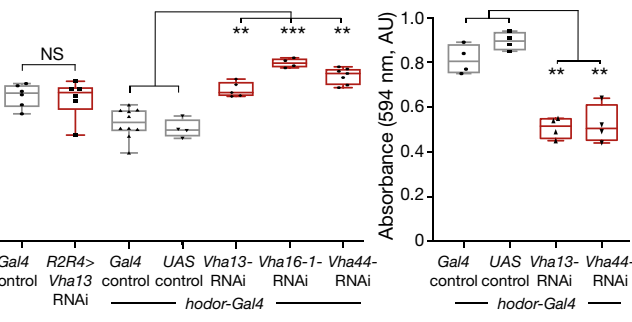

positive for LysoTracker (g) and Lamp1-mCherry (h) co-express Hodor in interstitial cells. Larvae were starved for $4 \mathrm{~h}$ for improved lysosomal visualization. i, A GFP-mCherry-Atg 8 a reporter reveals increased production of mCherry-positive autophagic punctae in interstitial cells; some are positive for GFP, the fluorescence of which is normally quenched under acidic conditions. Single confocal slices for each channel are shown below the three main panels. $\mathbf{j}, \mathbf{k}$, Knockdown of subunits of the V-ATPase complex from interstitial cells (using hodor-Gal4) but not from other enterocytes (using R2R4-Gal4) delays pupariation (j) and/or reduces food intake $(\mathbf{k})$. See Supplementary Information for sample sizes and full genotypes. Mann-Whitney $U$-tests or ordinary oneway ANOVA with Tukey post hoc tests were used for two-group comparisons, or for comparisons involving more than two groups, respectively. ${ }^{*} P<0.05$, ${ }^{* *} P<0.01,{ }^{* * *} P<0.001$. Box plots: line, median; box, 75 th -25 th percentiles; whiskers, minimum to maximum. Some images were false-coloured for consistency. N, nucleus. Scale bars, $30 \mu \mathrm{m}(\mathbf{e}) ; 10 \mu \mathrm{m}(\mathbf{f}-\mathbf{h}) ; 30 \mu \mathrm{m}(\mathbf{i}-\mathbf{k})$; $50 \mu \mathrm{m}(\mathbf{l})$.

zinc-elicited currents, but did result in currents with faster rise time and deactivation kinetics (Extended Data Fig. 6a-d), which supports the idea that zinc is a relevant Hodor ligand. On the basis of its sequence and conductance properties, Hodor has been proposed to transport chloride ${ }^{2,3}$, and the zinc-elicited currents that we observed in oocytes had a reversal potential that is consistent with chloride selectivity. In vivo experiments in flies showed that supplementation of a low-yeast diet with zinc led to a reduction of chloride levels in interstitial cells, whereas hodor mutation increased chloride levels (Fig. 3c, Extended Data Fig. 6g, h). Thus, Hodor is a pH-modulated, zinc-gated chloride channel.

We then sought to establish the relevance of zinc binding in vivo. We observed zinc enrichment in both the copper and iron cell regions of the larval gut (Extended Data Fig. 7a, b), revealing an unrecognized 
role for these Hodor-expressing regions in zinc handling. Mutation of hodor failed to affect this zinc accumulation, although dietary yeast levels did (Extended Data Figs. 5d, e, 7b, c), which is consistent with a role for Hodor in sensing rather than transporting zinc. (Notably, the white mutation-which is frequently used in the genetic background of Drosophila experiments-results in a small but significant reduction in both intestinal zinc accumulation and larval growth rate, although the status of the $w$ gene neither exacerbated nor masked the more substantial, hodor-induced developmental delay (Extended Data Fig. 7b-e, Supplementary Information)). Furthermore, larvae that were fed a low-yeast diet ate significantly more when the diet was supplemented with zinc; this effect was abrogated in hodor mutants (Fig. 3d). In a food choice experiment, control larvae developed a preference for zinc-supplemented food over time (Fig. 3e), which suggests that the preference develops after ingestion. Consistent with this idea, zinc preference was specifically abrogated in hodor mutants (Fig. 3e; we confirmed their general ability to discriminate between other diets (Extended Data Fig. 6f)). Thus, zinc sensing by Hodor is physiologically relevant in vivo. Metals such as zinc are primarily provided by yeasts in nature; Hodor may be one of several sensors used to direct larvae to nutrient-rich food sources.

\section{Cellular roles of a zinc-gated chloride channel}

The subcellular localization of Hodor suggests that it may normally maintain low cytoplasmic chloride concentrations by transporting it out of the interstitial cells and/or into their lysosomes. In accordance with this, and consistent with its putative lysosomal localization signals ${ }^{17}$, Hodor was specifically enriched in apical compartments containing late endosome or lysosomal markers, as well as decorating the brush border of interstitial cells (Fig. 3f-h, Extended Data Fig. 8a-e). The presence of Hodor in a subpopulation of lysosomes was of interest, because chloride transport across lysosomal membranes often sustains the activity of the proton-pumping vacuolar-type ATPase (V-ATPase) that maintains lysosomal acidity and Tor activation on the lysosome $\mathrm{e}^{18-20}$. To explore a role for Hodor in enabling Tor signalling, we tested whether the absence of hodor induced autophagy-a hallmark of reduced Tor signalling ${ }^{21}$. We first confirmed the induction of common autophagy markers in interstitial cells after genetic interference with the V-ATPase complex, which is known to promote autophagy by reducing lysosomal acidity and Tor signalling ${ }^{20,22}$ (Extended Data Fig. 9a,b). Similar to reduced V-ATPase function, loss of hodor increased autophagy in interstitial cells (Extended Data Fig. 9a). Expression of the dual autophagosome and autolysosome reporter UAS-GFP-mCherryAtg8a in the intestinal cells of hodor mutants confirmed the induction of autophagy (Fig. 3i), and revealed two additional features. First, the acidification of autophagic compartments was defective in hodor mutants (Fig. 3i, Extended Data Fig. 9c-e). Second, the increased autophagy and defective acidification observed in hodor mutants were particularly prominent in the two Hodor-expressing intestinal regions (the copper cell region and the iron cell region), consistent with cell-intrinsic roles for Hodor in these processes (Extended Data Fig. 9c, e). Additional support for the roles of lysosomal function and Tor signalling in controlling whole-body growth from interstitial cells was provided by the finding that most V-ATPase subunits were transcriptionally enriched in the copper cell region ('MidgutAtlas' RNA sequencing data ${ }^{12}$, confirmed with an endogenous protein reporter for the V-ATPase subunit Vha16-1, Extended Data Fig. 8f, g). Functionally, the downregulation of V-ATPase subunits specifically in Hodor-expressing cells-and not in other subsets of enterocytes, such as those targeted by R2R4-Gal $4^{23}$ (Extended Data Fig. 2h)-led to developmental delay and reduced food intake, phenotypes comparable to those observed as a result of hodor downregulation (Fig. $3 \mathrm{j}, \mathrm{k}$ ). Hence, although the directionality of zinc sensing and chloride transport in interstitial cells remains to be established, our data are consistent with roles for brush-border Hodor in transporting chloride out of interstitial cells-thus maintaining osmolarity and water balance. Lysosomal Hodor may transport chloride into the lysosome to sustain V-ATPase function, lysosomal acidification and TOR signalling, pointing to new links between lysosomal homeostasis in specialized intestinal cells, food intake and systemic growth (Extended Data Fig. 11). Nutrients such as amino acids are important regulators of Tor signalling ${ }^{21,24,25}$. Our genetic data are consistent with novel input from metals and/or micronutrients into Tor signalling. The nutrient-dependent zinc accumulation in lysosomal organelles-recently described in mammalian cells and nematode worms ${ }^{26,27}$-suggests that links between zinc, lysosomes and Tor may be of broader importance. Two attractive cell types in which to explore such links are the Paneth cells of the mammalian intestine, which accumulate zinc and regulate intestinal immunity and stem cell homeostasis ${ }^{28}$, and the lysosome-rich enterocytes' that have recently been described in fish and mice, which have roles in protein absorption ${ }^{29}$.

\section{Hodor as a target for disease vector control}

An extensive reconstruction of the hodor family tree supported the presence of a single member of the family in the ancestor of insects (Extended Data Fig. 10, Supplementary Information). Because Hodorlike proteins are present only in insects, they may prove to be highly specific targets for the chemical control of disease vectors, particularly given that mosquito genomes contain a single gene rather than the three paralogues that are found in most flies. To test this idea, we used CRISPR-Cas9 genome editing to generate a mutant that lacks the single hodor-like gene in the malaria vector Anopheles gambiae (AGAP009616) (Extended Data Fig. 10b, c, Supplementary Information). This gene is also expressed in the digestive tract-specifically in the midgut-and in Malphighian tubules ${ }^{30}$. Three independent deletion alleles revealed that $A G A P O 09616$ function is essential for the viability of $A$.gambiae (Extended Data Fig. 10d). A target that is expressed in the intestine, such as Hodor, is particularly attractive for vector control as it may circumvent accessibility issues and could be directly targeted using ingestible drugs such as those applied to larval breeding sites.

\section{Outlook}

Metals have received little attention in the contexts of development or whole-body physiology, and are often regarded as passive 'building blocks'. By revealing the roles of a metal sensor in food intake and growth control, our findings highlight the importance of investigating the instructive contributions of metals-and, more generally, micronutrients-to energy homeostasis. These mechanisms could prove to be useful in insect vector control.

\section{Online content}

Any methods, additional references, Nature Research reporting summaries, source data, extended data, supplementary information, acknowledgements, peer review information; details of author contributions and competing interests; and statements of data and code availability are available at https://doi.org/10.1038/s41586-020-2111-5.

1. Miguel-Aliaga, I. Nerveless and gutsy: intestinal nutrient sensing from invertebrates to humans. Semin. Cell Dev. Biol. 23, 614-620 (2012).

2. Feingold, D., Starc, T., O'Donnell, M. J., Nilson, L. \& Dent, J. A. The orphan pentameric ligand-gated ion channel $\mathrm{pHCl}-2$ is gated by $\mathrm{pH}$ and regulates fluid secretion in Drosophila Malpighian tubules. J. Exp. Biol. 219, 2629-2638 (2016).

3. Remnant, E. J. et al. Evolution, expression, and function of nonneuronal ligand-gated chloride channels in Drosophila melanogaster. G3 (Bethesda) 6, 2003-2012 (2016).

4. Colombani, J. et al. A nutrient sensor mechanism controls Drosophila growth. Cell 114, 739-749 (2003).

5. Géminard, C., Rulifson, E. J. \& Léopold, P. Remote control of insulin secretion by fat cells in Drosophila. Cell Metab. 10, 199-207 (2009).

6. Rodenfels, J. et al. Production of systemically circulating Hedgehog by the intestine couples nutrition to growth and development. Genes Dev. 28, 2636-2651 (2014). 


\section{Article}

7. Storelli, G. et al. Lactobacillus plantarum promotes Drosophila systemic growth by modulating hormonal signals through TOR-dependent nutrient sensing. Cell Metab. 14 403-414 (2011).

8. Waterhouse, D. F. \& Stay, B. Functional differentiation in the midgut epithelium of blowfly arvae as revealed by histochemical tests. Aust. J. Biol. Sci. 8, 253-277 (1955)

9. Poulson, D. F. \& Waterhouse, D. F. Experimental studies on pole cells and midgut differentiation in Diptera. Aust. J. Biol. Sci. 13, 541-567 (1960).

10. Dubreuil, R. R. et al. Mutations of a spectrin and labial block cuprophilic cell differentiation and acid secretion in the middle midgut of Drosophila larvae. Dev. Biol. 194, 1-11 (1998).

11. Li, H., Qi, Y. \& Jasper, H. Preventing age-related decline of gut compartmentalization limits microbiota dysbiosis and extends lifespan. Cell Host Microbe 19, 240-253 (2016).

12. Overend, G. et al. Molecular mechanism and functional significance of acid generation in the Drosophila midgut. Sci. Rep. 6, 27242 (2016)

13. Storelli, G. et al. Drosophila perpetuates nutritional mutualism by promoting the fitness of its intestinal symbiont Lactobacillus plantarum. Cell Metab. 27, 362-377.e8 (2018)

14. Filshie, B. K., Poulson, D. F. \& Waterhouse, D. F. Ultrastructure of the copper-accumulating region of the Drosophila larval midgut. Tissue Cell 3, 77-102 (1971).

15. Rulifson, E. J., Kim, S. K. \& Nusse, R. Ablation of insulin-producing neurons in flies: growth and diabetic phenotypes. Science 296, 1118-1120 (2002).

16. Dent, J. A. Evidence for a diverse Cys-loop ligand-gated ion channel superfamily in early bilateria. J. Mol. Evol. 62 523-535 (2006).

17. Negi, S., Pandey, S., Srinivasan, S. M., Mohammed, A. \& Guda, C. LocSigDB: a database of protein localization signals. Database (Oxford) 2015, bav003 (2015).

18. Sancak, Y. et al. Ragulator-Rag complex targets $\mathrm{mTORC1}$ to the lysosomal surface and is necessary for its activation by amino acids. Cell 141, 290-303 (2010).

19. Şentürk, M. et al. Ubiquilins regulate autophagic flux through mTOR signalling and ysosomal acidification. Nat. Cell Biol. 21, 384-396 (2019).
20. Zoncu, R. et al. mTORC1 senses lysosomal amino acids through an inside-out mechanism that requires the vacuolar $\mathrm{H}^{+}$-ATPase. Science 334, 678-683 (2011).

21. Valvezan, A. J. \& Manning, B. D. Molecular logic of mTORC1 signalling as a metabolic rheostat. Nat. Metab. 1, 321-333 (2019).

22. Mauvezin, C. \& Neufeld, T. P. Bafilomycin A1 disrupts autophagic flux by inhibiting both V-ATPase-dependent acidification and Ca-P6OA/SERCA-dependent autophagosomelysosome fusion. Autophagy 11, 1437-1438 (2015).

23. Hudry, B. et al. Sex differences in intestinal carbohydrate metabolism promote food intake and sperm maturation. Cell 178, 901-918.e16 (2019).

24. González, A. \& Hall, M. N. Nutrient sensing and TOR signaling in yeast and mammals. EMBO J. 36, 397-408 (2017)

25. Saxton, R. A. \& Sabatini, D. M. mTOR signaling in growth, metabolism, and disease. Cell 168, 960-976 (2017)

26. Blaby-Haas, C. E. \& Merchant, S. S. Lysosome-related organelles as mediators of metal homeostasis. J. Biol. Chem. 289, 28129-28136 (2014).

27. Roh, H. C., Collier, S., Guthrie, J., Robertson, J. D. \& Kornfeld, K. Lysosome-related organelles in intestinal cells are a zinc storage site in C. elegans. Cell Metab. 15, 88-99 (2012).

28. Holly, M. K. \& Smith, J. G. Paneth cells during viral infection and pathogenesis. Viruses 10, 225 (2018).

29. Park, J. et al. Lysosome-rich enterocytes mediate protein absorption in the vertebrate gut. Dev. Cell 51, 7-20.e6 (2019).

30. Baker, D. A. et al. A comprehensive gene expression atlas of sex- and tissue-specificity in the malaria vector, Anopheles gambiae. BMC Genomics 12, 296 (2011).

Publisher's note Springer Nature remains neutral with regard to jurisdictional claims in published maps and institutional affiliations.

(c) The Author(s), under exclusive licence to Springer Nature Limited 2020 


\section{Methods}

No statistical methods were used to predetermine sample size. The experiments were not randomized and the investigators were not blinded to allocation during experiments and outcome assessment.

\section{Fly husbandry}

Fly stocks were raised in incubators at $25^{\circ} \mathrm{C}, 65 \%$ humidity and on a $12 \mathrm{~h}: 12 \mathrm{~h}$ light:dark cycle, and were maintained on a standard cornmeal/ agar diet (6.65\% cornmeal, $7.1 \%$ dextrose, $5 \%$ yeast, $0.66 \%$ agar supplemented with $2.2 \%$ nipagin and 3.4\% propionic acid) ('high-yeast' food). For the 'low-yeast' food, all ingredients and quantities were the same as for the high-yeast food, except the yeast concentration was lower $(0.74 \%)$. All experiments were performed at $25^{\circ} \mathrm{C}$ or $29^{\circ} \mathrm{C}$. For experiments using $\mathrm{Gal} 8 \mathrm{O}^{\text {ts }}$, flies were initially raised at $18^{\circ} \mathrm{C}$ (permissive temperature), and were moved to $31^{\circ} \mathrm{C}$ (restrictive temperature) when Gal4 induction was required.

\section{Fly stocks}

The following fly stocks were used: hodor-Gal4 (ref. ${ }^{3}$ ), lab-Gal4 (Bloomington Drosophila Stock Center (BDSC): 43652), CtB-Gal4 (ref. ${ }^{31}$, gift from B. Denholm), Fer2LCH-Gal4 (DGGR: 113517), mex1-Gal4 (ref. ${ }^{32}$ ), Myo1A-Gal4 (Department of Drosophila Genomics and Genetic Resources (DGGR): 112001), R2R4-Gal4 (ref. ${ }^{23}$ ), tub-Gal8O ${ }^{\text {ts }}$ (BDSC: 7018), UAS-mCD8-GFP(BDSC: 5130), Fer1HCH-GFP (DGGR: 110620), UAS-Vha16-1-RNAi (GD17431, Vienna Drosophila Resource Center (VDRC): v49291), UAS-Vha44-RNAi (GD10617, VDRC: v46563), UASVha13-RNAi (GD10564, VDRC: v25985), UAS-nprl2-RNAi (KK101142, VDRC: v110579), UAS-iml-RNAi (KK101116, VDRC: v110386), UAS-hodorRNAi (KK106835, VDRC: v108337), UAS-hodor-RNAi \#2 (National Institute of Genetics (NIG):11340R-3), UAS-hodor (this study, see below for details), hodor $^{-1-}$ (this study, see below for details), UAS-Stinger-GFP (BDSC: 65402), UAS-shi ${ }^{K 4 A}$ (BDSC: 5811), KK control (VDRC: v60100), GD control (VDRC: v60000), UAS-Tor-RNAi (BDSC: 34639), UAS-Rheb (BDSC: 9688), UAS-S6K TE (BDSC: 6912), UAS-S6K ${ }^{\text {STDETE }}$ (BDSC: 6914), UAS$\operatorname{RagA}^{\text {T16N }}$;UAS-RagA ${ }^{\text {Q61 }}$;UAS-RagC $C^{S 54 N}$;UAS-RagC $C^{Q 99 L}$ (refs. ${ }^{33,34}$, gift from A. Teleman and C. Wilson), UAS-p62-GFP( ref. $^{35}$ ), Lamp1-mCherry (ref. ${ }^{36}$ ), hs-Ilp2 (ref. ${ }^{15}$ ), Vha16-1-GFP (DGGR: 110558), tub-Rab5-YFP, tub-Rab7YFP and tub-Rab11-YFP (ref. ${ }^{37}$, gifts from C. Wilson), UAS-ClopHensor (ref. ${ }^{38}$, gift from A. Rodan), UASp-GFP-mCherry-Atg8a (BDSC: 37749). Oregon $R(O r R)$ and $w^{1118}$ were used as control flies.

\section{Developmental rate, size and viability assays}

Enterocyte RNAi screen. UAS-RNAi lines for candidate genes were screened over three rounds to assess changes in developmental rate using two enterocyte Gal4 drivers (see Supplementary Information for a more detailed overview). Larvae were screened in batches of up to about 20 experimental crosses per diet per Gal4 driver, plus all appropriate controls. In the first two rounds, Gal4 and UAS parents were placed in experimental vials to seed them with test flies. Round two had a shorter laying period with more parent flies compared to round one, and flies were mated before addition to the experimental vial. At the midpoint of the laying interval, flies were considered to be 0 days of age. In the third round, eggs were laid over $24 \mathrm{~h}$ on egg-collection plates, then 50 eggs were transferred to a vial using moist filter paper. At this collection, flies were considered to be 0 days of age. For all of these protocols, pupae were counted every $24 \mathrm{~h}$ and the time to pupariation was calculated as a mean average for the vial.

Other experiments assessing developmental rate. Adult flies were allowed to lay eggs for $24 \mathrm{~h}$ at $25^{\circ} \mathrm{C}$ on apple juice plates containing a small amount of yeast paste. Embryos were collected and transferred to a new plate containing the appropriate diet, rinsing away yeast paste where necessary. After $4 \mathrm{~h}$ of hatching, first-instar larvae were seeded into vials containing the appropriate diet (close to the food) at 15-20 (or, in some experiments, 25) per vial, or onto plates at 50 per plate. At the midpoint of the hatching interval, flies were considered to be 1 day of age. Pupae were counted once in the morning and once in the evening and the time to pupariation was calculated as an average for the vial. For experiments requiring heat shock, control and experimental larvae were subjected to a temperature of $37^{\circ} \mathrm{C}$ twice a day for $45 \mathrm{~min}$ and then returned to either $25^{\circ} \mathrm{C}$ or $29^{\circ} \mathrm{C}$, as described previously ${ }^{15}$. For experiments assessing larval transitions and survival, seeded larvae were checked every $24 \mathrm{~h}$. Developmental stage was assessed on the basis of the size and maturity of their mouth hooks, and larvae were size-matched whenever appropriate.

Embryonic viability assays. Embryos on apple juice plates were collected after a 6-h egg-laying window at $25^{\circ} \mathrm{C}$. The number of hatched eggs and dead embryos were scored after $36 \mathrm{~h}$. Embryonic viability was calculated as the percentage of first-instar larvae divided by the total number of embryos (hatched larvae plus dead embryos).

Quantifications of pupal size. Pupae from different experimental conditions were collected and placed onto a coverslip and imaged using a Leica 10450528 camera attached to a Leica M165FC stereomicroscope using a $0.5 \times \mathrm{c}$-mount. Dimensions (length $(L)$ and width $(w)$ ) were measured with $\mathrm{Fiji}^{39}$. Pupal volume $(V)$ was calculated according to the following formula ${ }^{40}: V=4 / 3 \pi(L / 2)(w / 2) 2$. Each data point represents one pupal case.

\section{Immunohistochemistry}

Larvae of the appropriate developmental stage were selected, dissected in PBS, transferred to a polylysine slide and fixed with $4 \%$ formaldehyde (16\% formaldehyde (Thermo Fisher Scientific, 28908) diluted in PBS) for 20-40 min, depending on the specific antibody. Samples were washed with PBS, then PBT (PBS with $0.2 \%$ Triton X-100), and were blocked with PBTN (PBT with $4 \%$ normal horse serum) for $1 \mathrm{~h}$. Primary antibody was diluted in PBTN and was incubated with samples overnight at $4{ }^{\circ} \mathrm{C}$, and washed with PBT the next day. For certain antibodies, guts were either cut or holes were made in the sample to improve antibody penetration into the tissue. Fluorescently labelled secondary antibodies were then added for $1.5-5 \mathrm{~h}$ at room temperature or overnight at $4{ }^{\circ} \mathrm{C}$, and were washed away with PBT and PBS. For phalloidin staining, conjugated phalloidin in PBTN was added for $45 \mathrm{~min}$. Samples were then washed with PBS and mounted in Vectashield (with or without DAPI; Vector Labs, $\mathrm{H}-1200$ or $\mathrm{H}-1000$ respectively). Staining of experimental and control samples was carried out on the same slide to enable direct comparisons.

To visualize lipid droplets, fat body tissue surrounding the male gonad of second-instar larvae was dissected in PBS, mounted on polylysine slides and fixed for $30 \mathrm{~min}$. Samples were then washed three times in PBT and Nile Red stain (Thermo Fisher Scientific, N1142) was applied 1:500 for $30 \mathrm{~min}$ in the dark. Samples were then washed three times in PBS and mounted in Vectashield containing DAPI. Each data point corresponds to one dissected fat body from one larva (different data points correspond to different larvae).

For Lysotracker/Lysosensor stainings, guts were dissected in PBS and transferred to polylysine slides. Small punctures were made in the tissue using tungsten wire to enable the entry of LysoTracker Red DND-99 (Thermo Fisher Scientific, L7528) or LysoSensor Green DND189 (Thermo Fisher Scientific, L7535), which were applied at a 1:500 dilution in 4\% paraformaldehyde for 15-30 min (Fig. 3g) in the dark or imaged immediately under live conditions (Extended Data Fig. 9a). Samples were then washed with PBT and PBS, or blocked with PBTN, then immunostained. Samples were mounted in Vectashield.

For zinc staining, adult and larval guts were dissected in PBS, transferred to polylysine slides and fixed with $4 \%$ formaldehyde for $30 \mathrm{~min}$. They were then washed with ethanol, PBT and PBS. Guts were incubated with the zinc indicator FluoZin-3AM (1:3,000 in PBS containing 
$0.02 \%$ Triton and $0.001 \%$ Tween) at $38^{\circ} \mathrm{C}$ for $45 \mathrm{~min}$ in the dark. Guts were then washed with ethanol, PBT and PBS. Guts were mounted with Vectashield containing DAPI. To quantify zinc levels, integrated density was measured for the copper cell region using Fiji, ensuring that the area measured was the same between samples. Each data point corresponds to one gut.

For measurements of Ilp2 intensity in the insulin-producing cells of the brain, staining was performed as described above. After imaging, the freehand selection tool was used to draw around the insulinproducing cells on both sides of the brain, and the mean grey scale was calculated after subtracting from background staining.

For autophagy and lysosomal acidity quantifications using the dual UAS-GFP-mCherry-Atg8a reporter, the total number of punctae in each channel (GFP and mCherry) was separately counted by importing raw data into Fiji and using the 'find maxima' tool to highlight punctate structures. The same method was used to quantify Lysotracker, Lysosensor, p62 and Lamp-positive structures. For starvation experiments, larvae were placed in a moist clear dish for $7 \mathrm{~h}$ or overnight.

The following antibodies were used: rabbit anti-Hodor (1:500, this study), mouse anti- $\alpha$-Spectrin (1:10; DSHB, 3A9), anti-Ilp2 (1:200; gift from P. Léopold), p70 S6K (Thr398) (1:1,000; Cell Signaling, 9209S), pAKT (Ser505) (1:500; Cell Signaling, 4054), Akt (1:500; Cell Signaling, 9272S), tubulin (1:1,000; DSHB, 12G10). Conjugated fluorescent secondary antibodies (FITC-, Cy3- and Cy5-conjugated) were obtained from Jackson Immunoresearch and used at 1:200. Phalloidin conjugated to AlexaFluor647 or AlexaFluor488 was obtained from Thermo Fisher Scientific (A22287) and used at 1:100.

\section{Hodor antibody generation}

An antibody against Hodor was raised in rabbit by immunizing with a short peptide sequence found in the extra-cytoplasmic region of the protein (PVVHNKDGEEVP; amino acids 91-102). The antibody against Hodor was purified from the serum. This entire procedure was outsourced to New England Peptide.

\section{Assessments of midgut luminal acidity and diameter}

Larvae were selected on the basis of their developmental stage and placed on plates containing food supplemented with the $\mathrm{pH}$-sensitive dye $0.04 \%$ bromophenol blue (which changes from yellow at $\mathrm{pH} 3.0$ to blue at $\mathrm{pH}$ 4.6) for a minimum of $1 \mathrm{~h}$. Guts were dissected in unbuffered salt solution $(80 \mathrm{mM} \mathrm{NaCl}, 55 \mathrm{mM} \mathrm{KCl})$ and were immediately imaged using a Leica 10450528 attached to a Leica M165FC stereo microscope using a $0.5 \times \mathrm{c}$-mount. For measurements of gut diameter, guts were acquired as stated above and the diameter of the copper cell region was calculated using Fiji ${ }^{39}$.

\section{Food intake quantifications}

Larvae from seeded plates were matched for developmental stage (using mouth-hook anatomy) and size, and placed in plates containing $1 \%$ FCFblue dye for $45 \mathrm{~min}$. For diets supplemented with zinc, $\mathrm{ZnCl}_{2}$ (SigmaAldrich, Z0152) was used; larvae were raised on either supplemented or unsupplemented food and developmental experiments were performed side by side. Larvae were gently washed in distilled $\mathrm{H}_{2} \mathrm{O}$ to remove excess dye remaining on their outer cuticle, and were either imaged using a Leica DFC420C camera to visualize blue food in the gut, or placed in 2-mlEppendorf tubes containing $45 \mu \mathrm{l}$ distilled $\mathrm{H}_{2} \mathrm{O}$ and a 5 -mm ball bearing. These larvae were then homogenized twice for $60 \mathrm{~s}$ with a Qiagen Tissuelyser II at $30 \mathrm{~Hz}$ and then centrifuged for $60 \mathrm{~s}$ at $13,000 \mathrm{~g}$. The dye content of the supernatant was measured at $594 \mathrm{~nm}$ either using a NanoDrop ND-1000 spectrophotometer or with a FLUOstar Omega microplate reader.

For mouth-hook contraction assays, larvae were placed on apple juice plates covered with a thin layer of yeast paste. Larvae were then given 5 min to adjust to their new environment and mouth-hook contractions were counted for $30 \mathrm{~s}^{41,42}$. This value was multiplied by 2 to obtain counts per minute.

\section{Food preference experiments}

Developmentally matched first-instar larvae were raised on low- or high-yeast food and were starved for $3 \mathrm{~h}$. They were then placed onto a choice assay plate containing an agar separator with two sources of food on either side, as described previously ${ }^{43}$, so that they had a choice between high- and low-yeast, or zinc-supplemented and nonzinc-supplemented low-yeast food. $\mathrm{ZnCl}_{2}$ (Sigma \#Z0152) was used to supplement low-yeast food to assess zinc preference. $\mathrm{ZnSO}_{4}$ supplementation also elicited preference (data not shown). The number of larvae on each side of the plate (and on the agar) was scored at the designated time points, and was used to calculate a preference index (PI) as follows ${ }^{44}$ :

$$
\frac{N_{\mathrm{x}} /\left(N_{\mathrm{x}}+N_{\mathrm{a}}+N_{\mathrm{l}}\right)}{N_{\mathrm{l}} /\left(N_{\mathrm{x}}+N_{\mathrm{a}}+N_{\mathrm{l}}\right)}
$$

in which $N_{\mathrm{x}}=$ number of larvae that preferred food x (x could be $\mathrm{ZnCl}_{2}$ or $\mathrm{ZnSO}_{4}$-supplemented low-yeast diet, or a high-yeast diet), $N_{1}=$ number of larvae that preferred a low-yeast diet and $N_{\mathrm{a}}=$ number of larvae with no preference.

$\log _{2}$ was then applied to the $\mathrm{PI}_{\mathrm{x}}$. If $\log _{2}\left(\mathrm{PI}_{\mathrm{x}}\right)>0$, it means larvae prefer food $x$ to low-yeast food; if $\log _{2}\left(\mathrm{PI}_{\mathrm{x}}\right)<0$, it means larvae prefer low-yeast food to food $x$. Loess analysis was then performed to fit the data across the time points using ggplot 2 geom_smooth() function with argument method = "loess" and se = "TRUE".

\section{Electron microscopy}

First-instar larval guts were dissected and fixed in $2.5 \%$ glutaraldehyde in $\mathrm{PB}(0.1 \mathrm{M}$ phosphate buffer, $\mathrm{pH}$ 7.2), followed by fixation in $1 \%$ osmium tetroxide $+1.5 \%$ potassium ferrocyanide for 60 min at $4{ }^{\circ} \mathrm{C}$. After dehydration with ethanol, guts were infiltrated and embedded in Durcupan, and ultra-thin (70-nm) sections were cut using a Leica Ultracut UCT. Ultra-thin sections were contrasted with $2 \%$ uranyl acetate for $10 \mathrm{~min}$ and lead citrate for $5 \mathrm{~min}$. They were then analysed using a Morgagni 268 TEM (80-kV) electron microscope (FEI) and imaged using a sideentry Morada CCD Camera (EMSIS).

\section{Image acquisition and processing}

All fluorescent images were acquired using a Leica SP5 II confocal microscope and Leica LAS AF software. The same confocal settings, including laser power, were applied to both experimental and control groups. Images were processed using $\mathrm{Fiji}^{39}$. All statistical analyses were performed on raw images. To visualize the whole gut, images were stitched together using the Pairwise Stitching plugin ${ }^{45}$. For cell-volume measurements, images of midgut copper cell regions were imported into IMARIS 9.2.1 and cell volume was calculated by measuring greenlabelled interstitial cells in 5- $\mu \mathrm{m}$ intervals. For subcellular localization experiments (for example, Hodor, Lysotracker Lamp1 or Rab5, 7, 11), the number of YFP and/or magenta-positive punctae were counted in a single plane of an interstitial cell (total 3 cells per gut) and the percentage of YFP-positive punctae that were also positive for Hodor antibody signal was calculated. Some images were false-coloured for consistency with other images in the manuscript.

\section{Quantitative PCR with reverse transcription}

For each sample, RNA was extracted from 15 whole larvae (L2) using Trizol (Invitrogen), and cDNA was synthesized using iScript cDNA synthesis kit (Bio-Rad, 170-8890) from 500 ng of total RNA. Quantitative PCR was performed by mixing cDNA sample ( $5 \mathrm{ng}$ ) with iTaq Universal SYBR Green Supermix (Bio-Rad, 172-5124) and the optimized primer pairs (see below). Expression values were normalized to gapdh. For each gene at least three independent biological replicates were used, and two technical replicates were performed. The primer pairs were as follows: hodor: forward primer, GAACACCACGGATGCTTTCAG; reverse 
primer, ATGGACTCTGCGTTTTTCAGC.gadph: forward primer, CATT GTGGGCTCCGGCAA; reverse primer, CGCCCACGATTTTCGCTATG.

\section{Western blot analyses}

For the pAkt western blots, extracts of second-instar larvae were prepared by mechanical homogenization and lysis in RIPA buffer (Thermo Fisher, 89900) with complete protease inhibitor cocktail (Roche, 11836170001) and phosphatase inhibitors (Sigma-Aldrich, 4906837001). Sixty larvae for each treatment group were pooled, and each experiment was repeated at least once. Lysates were cleared from debris and lipids by centrifugation for $10 \mathrm{~min}$ in a table-top centrifuge at $4{ }^{\circ} \mathrm{C}$. Total protein concentrations were determined using the Pierce BCA Protein Assay kit (Thermo Fisher, 23227) and concentrations of lysates were adjusted accordingly. For the pS6K western blots, larvae were directly lysed in $1 \times$ Laemli containing protease and phosphatase inhibitors. Lysates were cleared from debris by a 10-min centrifugation at $4{ }^{\circ} \mathrm{C}$. Samples were boiled, resolved on SDS-PAGE, and transferred by standard protocols.

\section{ClopHensor experiments}

For ClopHensor experiments, first-instar larval intestines from the relevant genotypes were dissected in Drosophila saline, consisting of $117.5 \mathrm{mM} \mathrm{NaCl}, 20 \mathrm{mM} \mathrm{KCl}, 2 \mathrm{mM} \mathrm{CaCl}_{2}, 8.5 \mathrm{mM} \mathrm{MgCl}_{2}, 10.2 \mathrm{mM} \mathrm{NaHCO}_{3}$, $4.3 \mathrm{mM} \mathrm{NaH}_{2} \mathrm{PO}_{4}, 15 \mathrm{mM}$ HEPES and $20 \mathrm{mM}$ glucose, $\mathrm{pH}$ 7.0. They were mounted on polylysine slides and bathed in standard bathing medium consisting of a 1:1 mix of Drosophila saline and Schneider medium (Thermo Fisher Scientific, 21720024). Intestines were then imaged live using a Leica SP5 Inverted microscope with excitation set at 488 $\mathrm{nm}$ (green emission), $458 \mathrm{~nm}$ (cyan emission) and $543 \mathrm{~nm}$ (red emission). Pixel intensity for the upper and lower limits of the whole copper cell region (avoiding the section in which the gut lumen is visible) was measured, and the ratio between fluorescence intensity at $488 \mathrm{~nm}$ and $458 \mathrm{~nm}$ was used to calculate $\mathrm{pH}$, while the $458 \mathrm{~nm} / 543 \mathrm{~nm}$ ratio was used for intracellular chloride measurements. For chloride calibration, larval intestines were dissected in Drosophila saline and then bathed in chloride calibration solution, consisting of $\mathrm{NaCl}$ (varying amounts), Na-gluconate (varying amounts), $50 \mathrm{mM} \mathrm{K-gluconate,} 2 \mathrm{mM} \mathrm{Ca-glu-}$ conate, $8.5 \mathrm{mM}$ Mg-gluconate, $20 \mathrm{mM}$ glucose, $15 \mathrm{mM}$ HEPES pH 7.2, $10 \mu \mathrm{M}$ tributyltinchloride (Sigma-Aldrich), $5 \mu \mathrm{M}$ nigericin (Invitrogen), $5 \mu \mathrm{M}$ carbonyl cyanide 3-chlorophenylhydrazone (Sigma-Aldrich) and $5 \mu \mathrm{M}$ valinomycin (Sigma-Aldrich). Intestines were allowed to equilibrate for $1 \mathrm{~h}$ in their respective solutions before imaging using a Leica SP5 Inverted microscope. The ratio of fluorescence intensity at $458 \mathrm{~nm} / 543 \mathrm{~nm}$ for each chloride concentration was interpolated as a sigmoidal curve using a logistic dose-response sigmoidal fit function in Prism.

\section{Microbiome experiments}

Bacterial strains and growth conditions. We used Acetobacter pomorum $^{\mathrm{WJL}}\left(\right.$ ref. $^{46}$ ) and Lactobacillusplantarum $^{\mathrm{NC} 8}\left(\right.$ ref. $\left.^{47}\right)$. . . pomorum was grown in Mannitol Broth (Bacto peptone $3 \mathrm{gl}^{-1}$, yeast extract $5 \mathrm{gl}^{-1}$, D-mannitol $25 \mathrm{gl}^{-1}$ ) for $24 \mathrm{~h}$ at $30^{\circ} \mathrm{C}$ under $180 \mathrm{rpm}$ agitation. L. plantarum was grown in MRS Broth (Carl Roth) at $37^{\circ} \mathrm{C}$ overnight without agitation.

Germ-free flies. Flies were rendered germ-free following the protocol described previously ${ }^{48}$. Germ-free flies were maintained on fly medium supplemented with antibiotics: kanamycin $50 \mu \mathrm{g} \mathrm{ml}^{-1}$ (Sigma-Aldrich, K1377), ampicillin $50 \mu \mathrm{g} \mathrm{ml}^{-1}$ (Sigma-Aldrich, A0166), tetracycline $10 \mu \mathrm{g} \mathrm{ml}^{-1}$ (Sigma-Aldrich, T7660), erythromycin $5 \mu \mathrm{g} \mathrm{ml}^{-1}$ (SigmaAldrich, E5389). Axenicity was confirmed by crushing the flies and plating the lysate on LB Agar (Carl Roth) and MRS Agar (Carl Roth) plates.

Developmental timing. Larvae mono-associated with $A$. pomorum were reared on a medium composed of agar $\left(7.14 \mathrm{gl}^{-1}\right)$, cornmeal $\left(80 \mathrm{~g} \mathrm{l}^{-1}\right)$, yeast (50 $\mathrm{gl}^{-1} \mathrm{or} \mathrm{gl}^{-1}$ for rich (high-yeast) and poor (low-yeast) medium, respectively), sucrose $\left(45 \mathrm{gl}^{-1}\right)$, nipagin $\left(0.7 \mathrm{gl}^{-1} ;\right.$ Sigma-Aldrich, 85265$)$ and propionic acid (0.1\%; Sigma-Aldrich, P5561). Germ-free larvae and larvae mono-associated with $L$. plantarum were reared on a medium composed of agar $\left(7.1 \mathrm{gl}^{-1}\right)$, cornmeal $\left(80 \mathrm{gl}^{-1}\right)$, yeast $\left(50 \mathrm{gl}^{-1}\right.$ or $7 \mathrm{gl}^{-1}$ for rich and poor medium, respectively), nipagin $\left(5.2 \mathrm{gl}^{-1}\right)$ and propionic acid $(0.4 \%)$. Germ-free flies were allowed to lay eggs in sterile breeding cages overnight. Germ-free embryos were collected and transferred in groups of 40 into fresh sterile tubes. Bacterial cultures were washed in PBS and inoculated on the eggs at a final concentration of around $10^{7}$ colony-forming units (cfu) per tube for $A$. pomorum and around $10^{8}$ cfu per tube for $L$. plantarum. Tubes were kept at $25^{\circ} \mathrm{C}$ and the number of newly emerged pupae was scored every day until all pupae had emerged.

Bacterial loads. Larvae bi-associated with A. pomorum and $L$. plantarum were reared on a medium composed of agar $\left(7.14 \mathrm{gl}^{-1}\right)$, cornmeal $\left(80 \mathrm{gl}^{-1}\right)$, yeast $\left(50 \mathrm{gl}^{-1}\right)$, sucrose $\left(45 \mathrm{gl}^{-1}\right)$, nipagin $\left(0.7 \mathrm{gl}^{-1} ;\right.$ Sigma-Aldrich, 85265$)$ and propionic acid (0.1\%; Sigma-Aldrich, P5561). Germ-free flies were allowed to lay eggs in sterile breeding cages overnight. Germ-free embryos were collected and transferred in groups of 40 into fresh sterile tubes. Bacterial cultures were washed in PBS and mixed together before inoculation on the eggs, yielding an initial concentration of $5 \times 10^{7} \mathrm{cfu}$ per tube for L. plantarum and around $5 \times 10^{6} \mathrm{cfu}$ per tube for $A$. pomorum. Size-matched third-instar larvae were collected, surface-sterilized in $70 \%$ ethanol and placed in microtubes containing $400 \mu \mathrm{l} \mathrm{PBS}$ and 0.75-1 mm glass microbeads (Carl Roth, A554.1). Larvae were then homogenized using a Precellys 24 Tissue Homogenizer (Bertin Technologies). Lysate dilutions were plated using an EasySpiral automatic plater (Intersciences), on MRS Agar with selective antibiotics to select L. plantarum and A. pomorum; kanamycin $\left(50 \mu \mathrm{g} \mathrm{ml}^{-1}\right)$ enabled selective growth of $L$. plantarum and ampicillin $\left(10 \mathrm{gl}^{-1}\right)$ enabled selective growth of A. pomorum. Plates were incubated at $30^{\circ} \mathrm{C}$ for $48 \mathrm{~h}$ for $A$. pomorum and $37^{\circ} \mathrm{C}$ for $24 \mathrm{~h}$ for $L$. plantarum, and colonies were counted using an automatic colony counter Scan1200 (Intersciences).

\section{Wing size measurements}

The wings of 3-5 day old adult flies were dissected in isopropanol and mounted on a slide. The excess isopropanol was wiped off and several drops of Euparal (Anglian Lepidopterist Supplies, DS31) was added to the slide; a coverslip was placed on top. The slides were incubated at $60^{\circ} \mathrm{C}$ overnight and imaged using a Leica 10450528 attached to a Leica M165FC stereo microscope. To quantify wing size, a straight line was drawn from the distal tip of the L3 vein to the proximal tip of the L4 vein using Fiji.

\section{Experimental design and statistical analyses}

Sample sizes were not limiting and were chosen empirically on the basis of the variability of each scored phenotype. Comparable sample sizes for each genotype/condition were used in every experiment. For sample size information (repeats, number of flies and so on), see Supplementary Information. All experiments were performed at least three times yielding comparable outcomes. Further replicates were included if necessary, for example to account for variability resulting from incubator temperature fluctuations or food-batch variation. Experimental and control flies were bred in identical conditions, and were randomized whenever possible (for example, with regard to housing and position in tray). Control and experimental samples were dissected and processed at the same time and on the same slides. The experimenter was typically not blind to the genotypes and conditions.

Data analysis was carried out in Prism 7. For comparisons involving two groups, a non-parametric Mann-Whitney $U$-test was used. For cases in which more than two groups were compared, an ordinary one-way ANOVA test was performed with a Tukey post hoc test. For phenotypes for which a batch effect was apparent (for example, day of experiment), a two-way ANOVA was used. Significance values are denoted as follows: ${ }^{*} P<0.05,{ }^{* *} P<0.01,{ }^{* * *} P<0.001$. Data are displayed as box plots 
with line, median; box, 75th-25th percentiles; and whiskers, minimum to maximum.

\section{Smurf assays}

These were conducted by adapting the adult protocol described previously ${ }^{49}$. In brief, experimental larvae were removed from seeded plates and washed in distilled $\mathrm{H}_{2} \mathrm{O}$. They were then placed onto low-yeast food containing $1 \%$ FCF blue dye and allowed to feed overnight. The next day, larvae were washed and imaged using a Leica DFC420C camera attached to a Leica M165FC stereo microscope.

\section{Triacylglyceride quantification}

Triacylglycerides were quantified in whole second-instar larvae as previously described ${ }^{50}$. In brief, five second-instar larvae were pooled per sample, weighed and then homogenized in PBS $+0.05 \%$ Tween. Samples were heated for $5 \mathrm{~min}$ at $70^{\circ} \mathrm{C}$ to inactivate lipases, and were then centrifuged to remove debris. A $20-\mu$ l portion of supernatant for each sample was added to $200 \mu \mathrm{l}$ of Thermo Infinity Triglyceride Reagent (Thermo Scientific, TR22421) in duplicates, which was then incubated for $10 \mathrm{~min}$ at $37^{\circ} \mathrm{C}$. Absorbance was measured at $540 \mathrm{~nm}$ using a plate reader, and triacylglyceride levels for each duplicate were averaged and corrected for weight.

\section{Hodor mutant generation}

A hodor mutant line was generated as described previously ${ }^{51}$. Homology arms $5^{\prime}$ and $3^{\prime}$ to the hodor gene were amplified from $w^{1118}$ DNA using primers $\mathrm{HA} 5 \mathrm{~F} / \mathrm{R}$ and $\mathrm{HA} 3 \mathrm{~F} / \mathrm{R}$ (see below). The PCR products were cloned into pTV Cherry using NotI and Acc651 (New England Biolabs (NEB), R0189 and R0599, respectively) or Ascl and Spel (NEB, R0558 and R3133, respectively) restriction enzymes. The completed pTV Cherry vector with both homology arms was amplified, purified and injected into $y w$ embryos with 'Delta 2-3' Helper DNA (injections performed by Drosophila Transgenesis Service, Universidad Autonoma de Madrid) to randomly integrate into the genome by P-element insertion. Transformants were crossed to $h s-F L P, h s-I-S c e l$ flies and larval progeny were heat-shocked to induce homologous recombination. Mottled eyed adults were collected and crossed to ubiquitin-Gal4[3xP3$G F P]$. Progeny from this cross were screened for the presence of redeyed individuals, indicative of a successful recombination event. The ubiquitin-Gal4[3xP3-GFP] was later removed by selecting against the presence of GFP in the ocelli. The following primers were used: HA3 F/ R: forward, ACTAGTGTTCGTCAGGGAAAGAGAGCCATTC; reverse, GGCGCGCCTCCCATCATTGTTAACTCAAC. HA5 F/ R: forward, GC GGCCGCAGACGCTTGCCAACGATTAAGTACC; reverse, GGTACCGAA TCACGGGACTCAGTGGGTAAGTTTTCAGGAG.

\section{Generation of UAS-hodor}

To overexpress hodor, hodor cDNA was amplified from adult Oregon- $R$ gut RNA using the primers Hodor F and Hodor R. The PCR product was digested with Notl and EcoRI (Promega, R6435 and R6017, respectively) and cloned into the $p U A S T a t t B$ vector ${ }^{52}$. hodor-containing pUASTattB was amplified, purified and then injected into $\mathrm{ZH}$-attP-22A embryos ${ }^{52}$, which have an attP site on chromosome $2 \mathrm{~L}$ (injections were carried out by Drosophila Transgenesis Service, Universidad Autonoma de Madrid). Injected flies were crossed to $w^{1118}$ and progeny were screened for orange eyes, indicative of successful transgenesis. The following primers were used:Hodor F/R: forward,CAACGACGTGCAAGACATGACTAAC; reverse, GCTCTAGGATCACAGAATGGCTCTC.

\section{Modelling of Hodor structure and zinc binding}

The three-dimensional structure of Hodor was predicted using homology modelling by templating the sequence on to the pentamer (Protein Data Bank (PDB) ID:5VDI). Potential zinc $\left(\mathrm{Zn}^{2+}\right)$-binding sites were predicted with the MIB: Metal Ion-Binding Site Prediction and Docking software using the fragment transformation method ${ }^{53}$. The residue pairs with the highest predicted binding score were E255 and E296; C207 and C221; and H94 and D97. The top three binding sites were used to seed mutational binding-affinity calculations. Models of all possible single $(120)$ and double $(2,340)$ mutants of the three binding sites were prepared. Structure refinement was performed with 2,000 steps of conjugate gradient and steepest descent energy minimization with a $2 \mathrm{kcal} \mathrm{mol}^{-1}$ restraint on peptide backbone atoms, using the AMBER ff14SB force-field ${ }^{54}$. The structural stability and zinc binding affinity were calculated using the molecular-mechanics Poisson-Boltzmann surface area (MMPBSA) method ${ }^{55}$.

\section{Electrophysiology of Xenopus oocytes}

cRNA synthesis. hodor cDNA was PCR-amplified from Canton S flies using the primers below, which introduced Xbal and NotI sites. The PCR product was digested with Xbal and NotI and ligated into pGH19 vector (a derivative of $\mathrm{pGEMHE}^{56}$ ). This vector was linearized using NotI-HF (NEB, R3189S) for $2 \mathrm{~h}$ at $37^{\circ} \mathrm{C}$. The linearized DNA was purified using a PCR purification Kit (Qiagen, 28104) and eluted in $30 \mu$ l RNase-free water. RNA synthesis was performed with approximately $1 \mu \mathrm{g}$ DNA using mMessage mMachine T7 Transcription Kit including 15 min of DNase treatment (Ambion, AM1344). RNA was treated with a Zymo Clean \& Concentrator Kit (Zymo, R1013) and aliquoted at a concentration of approximately $1 \mu \mathrm{g}^{-1}$ for injection. The following primers were used: cRNA: forward, GATCTCTAGACAAGACATGACTAACCACC; reverse, CTAGGCGGCCGCCTCAAAGGCAGTAGACCAGG.

Oocyte preparation. Xenopus laevis ovaries (Nasco) were dissected and dissociated by incubating in $\mathrm{Ca}^{2+}$-free ND96 saline $(96 \mathrm{mM} \mathrm{NaCl}, 2 \mathrm{mM}$ $\mathrm{KCl}, 5 \mathrm{mMHEPES}, 3 \mathrm{mM} \mathrm{MgCl}_{2}$, adjusted to $\mathrm{pH} 7.4$ with $\mathrm{NaOH}$ ) containing 50-60 mg Type2 collagenase (lot-dependent) (Worthington LS004176), $25 \mathrm{mg}$ BSA (Sigma-Aldrich, A3311) and $12.5 \mathrm{mg}$ trypsin inhibitor (from chicken egg white; Sigma, T9253) for 90-120 min. Dissociated oocytes were then washed in $\mathrm{Ca}^{2+}$-free ND96 and manually selected into Barth's medium $\left(88 \mathrm{mM} \mathrm{NaCl}, 1 \mathrm{mM} \mathrm{KCl}, 0.33 \mathrm{mMCa}\left(\mathrm{NO}_{3}\right)_{2}, 0.41 \mathrm{mMCaCl}_{2}, 0.82\right.$ $\mathrm{mM} \mathrm{MgSO}_{4}, 2.4 \mathrm{mM} \mathrm{NaHCO}_{3}, 5 \mathrm{mM} \mathrm{HEPES}$ and $0.1 \mathrm{mg} \mathrm{ml}^{-1}$ gentamycin, $\mathrm{pH} 7.6$ with $\mathrm{NaOH}$ ) for injection the following day. Oocytes were injected with 50 ng RNA 24-36 h before recording using Nanoject III (Drummond Scientific) and kept in Barth's medium at $17^{\circ} \mathrm{C}$ until recording.

Recordings. Two-electrode voltage recordings were carried out at room temperature with an Oocyte Clamp OC-725C amplifier (Warner Instruments) and digitized using a Digidata $1550 \mathrm{~B}$ (Axon Instruments) interface and pClamp 11 software. Data were filtered at $1 \mathrm{kHz}$ and sampled at $10 \mathrm{kHz}$. Recordings were performed using borosilicate glass pipettes with resistances of around $1 \mathrm{M} \Omega$ when filled with $3 \mathrm{M} \mathrm{KCl} . \mathrm{ZnCl}_{2}$ (Sigma-Aldrich, Z0152) was diluted into a standard ND96 extracellular solution ( $96 \mathrm{mM} \mathrm{NaCl}, 2 \mathrm{mM} \mathrm{KCl}, 5 \mathrm{mM} \mathrm{HEPES}, 1 \mathrm{mM} \mathrm{MgCl} 2,2 \mathrm{mM}$ $\mathrm{CaCl}_{2}$ adjusted to $\mathrm{pH} 7.4$ with $\mathrm{NaOH}$ ). Current-voltage relationships were obtained using $200 \mathrm{~ms}$ voltage ramps from $-120 \mathrm{mV}$ to $120 \mathrm{mV}$ applied every $500 \mathrm{~ms}$ with an inter-stimulus holding potential of $-80 \mathrm{mV}$. Dose-response relationships were calculated using peak currents measured at $100 \mathrm{mV}$ and normalized to maximal currents elicited in response to $1 \mathrm{mM} \mathrm{ZnCl}_{2}$. Activation and deactivation kinetics were determined by fitting the rising and decaying phases of zinc-activated currents with single exponentials. Data were analysed in Clampfit 11 (Molecular Devices) and visualized with $\mathrm{R}$ ( $\mathrm{R}$ version 3.5.1).

\section{Phylogenetic analyses}

hodor orthologue identification, alignment, and phylogenetic reconstruction. To retrace the evolutionary history of hodor, we first queried OrthoDB v9 to identify gene family members of hodor and its two paralogues in D. melanogaster (FBgn0029733, FBgn0036727). As our intention was to characterize the emergence of hodor rather than build a comprehensive tree that included evolutionarily distant orthologues, analysis was restricted to the Arthropoda 
(EOG090X08ZM), which in OrthoDB v9 principally covers insect species. This enabled careful manual curation, as detailed below. We first retrieved coding sequences (CDS) corresponding to the proteins in EOG090X08ZM from the relevant source databases. Orthologue identification relies on single protein sequence per gene. As most metazoan genes have multiple splice isoforms, that single, often arbitrary sequence need not be the most suitable for comparison against a given focal sequence of interest (here hodor). To reduce alignment errors and provide maximum coverage of regions orthologous to the focal hodor protein sequence, we therefore systematically surveyed protein isoforms and swapped the CDS in EOG090X08ZM for a more suitable isoform if available as follows: for Ceratitis capitate, we swapped in XM_004527025.1 for CCAP005795; for Drosophila grimshawi, we swapped in Dgri $\backslash$ GH17038-PB for Dgri $\backslash G H 17038$, and Dgri $\backslash G H 15188$ PB for Dgri $\backslash \mathrm{GH} 15188$; for Bactrocera dorsalis, we swapped in 84262 for 1780586 and 244888 for 11780102 (as provided by i5k); for Dyak\ GE19913 in Drosophila yakuba, the sequence in the source database (FlyBase) is 1nt too long-a C is inserted at position 109. This $\mathrm{C}$ was removed to make the CDS length consistent with the protein length. The resulting sequence has a predicted internal stop codon, which may be a sequencing error. For Megaselia scalaris, all proteins from this species were removed as they are partially unresolved. For Drosophila suzukii, all proteins from this species were removed. Annotated protein lengths are quite different from all other Drosophila spp., suggesting potential gene prediction issues.

After manual curation, the surviving set of 109 proteins were aligned using mafft-linsi with default settings and alignments back-converted into CDS. The nucleotide-level alignment was then used to build a phylogenetic tree using RaxML v8.1.16 with the following parameters: -f a -x 12345 -p 12345 -\# 1000 -m GTRGAMMA.

Subsequent tree exploration highlighted $C$. capitata as having only two paralogues in the EOG090X08ZM set where three would have been expected. A tblastn query of the annotated $C$. capitata transcriptome using hodor revealed three bona fide hits, one of which (LOC101460849) was missing from EOG090X08ZM. Alignment and tree building were therefore repeated after inclusion of a reconstructed CDS from this locus and the final tree was rooted in accordance with results from a previous phylogenomic analysis of insects ${ }^{57}$. There are 110 proteins in the final dataset.

Trees were rendered with FigTree v1.4.2 (http://tree.bio.ed.ac.uk/ software/figtree/) and Adobe Illustrator.

Testing for purifying, relaxed, and positive selection. Data on single nucleotide polymorphisms (SNPs) in hodor were retrieved from the PopFly genome browser (http://popfly.uab.cat/) ${ }^{58}$. Within-species diversity at non-synonymous $(\mathrm{pN})$ and synonymous $(\mathrm{pS})$ sites was then calculated globally (across all D. melanogaster populations) and for a defined high-diversity population (ZI, Zimbabwe) using the seqinr $\mathrm{R}$ package $\mathrm{e}^{59}$. Pairwise rates of non-synonymous $(\mathrm{dN})$ and synonymous (dS) divergence between hodor and its orthologues in other Drosophila species (as depicted in Extended Data Fig. 10 and Supplementary Fig. 2) were calculated using a relevant method ${ }^{60}$, implemented in the PopGenome R package ${ }^{61}$.

To test for positive, relaxed and purifying selection in phylogenetic framework, we made use of a collection of likelihood ratio tests provided by the Datamonkey Adaptive Evolution Server (https://www.datamon key.org/). In particular, we tested for relaxed selection using the RELAX statistical framework ${ }^{62}$, comparing rates of evolution in the hodor family clade (purple box in Extended Data Fig. 10a and Supplementary Fig. 2) with the two clades (grey boxes) containing hodor paralogues.

\section{Mosquito strains and rearing}

Mosquitoes were reared under standard conditions at $27^{\circ} \mathrm{C}$ and $80 \%$ relative humidity with access to fish food as larvae and $5 \%$ glucose solution as adults. The mosquito strain used in this project, the $A$.gambiae
$\mathrm{G} 3$, is reasonably amenable to rearing and microinjection. We obtained this line from the MR4 (MRA-112) and it was originally isolated from west Africa (MacCarthy Island, The Gambia) in 1975 (Malaria Research \& Reference Reagent Resource Center). Cas9 mosquitoes were generated previously ${ }^{63}$ using human-codon optimized SpCas $9^{64}$ (https://www. addgene.org/42230/) under control of the vasa promoter within the pDSAY vector ${ }^{65}$ and inserted at the X locus (2L:10526503). The protocols and procedures used in this study were approved by the Animal Ethics Committee of Imperial College and are in compliance with United Kingdom Home Office regulations.

\section{Identification of the A.gambiae hodor gene}

AGAP009616 is the predicted one-to-many orthologue of Drosophila Hodor-family proteins ${ }^{66}$. To confirm this, the full-length protein sequence of $D$. melanogaster Hodor was used for tBLASTN searches of the A. gambiae genome transcript gene set (AgamP4.10) using VectorBase BLAST (https://www.vectorbase.org). Top-ranking hits were manually searched and AGAP009616 was determined as the highest ranking candidate, with $48.4 \%$ overall identity. No sequence similarity was detected between the predicted coding part of exon 1 of the annotated AGAPO09616 transcript and Drosophila hodor or its orthologues from more distantly related Aedes or Culex mosquito species. Although we found evidence for the existence of upstream exon 1 using RNA-seq BAM alignment files from $\mathrm{G} 3$ adult females (T. Nolan, unpublished data) visualized by IGV software ${ }^{67}$, predictions regarding the structure of exon 1 differ between members of the Anopheles genus. For this reason, the conserved exon 2 was chosen as the target for Cas 9 genome editing.

\section{Protein sequence alignments}

Protein sequence alignments were generated using Clustal Omega 1.2.3 using default parameters (https://www.ebi.ac.uk/Tools/msa/ clustalo ${ }^{68}$ ) and were visually modified using ESPript 3.0 to highlight percentage equivalence between sequences (http://espript.ibcp.fr/ ESPript/ESPript $/{ }^{69}$ ).

\section{Generation of transgenic guide RNA mosquito strains}

To generate CRISPR guide RNA (gRNA) germline transformation constructs, a single gRNA target site was identified within the second exon of $A G A P 009616$ and assessed for potential off-targets using flyCRISPR (http://targetfinder.flycrispr.neuro.brown.edu/) and ZiFIT (http://zifit. partners.org/). Because predictions regarding the structure of exon 1 differed between the closely related $A$.gambiae, Anopheles coluzzi and A. gambiae Pimperena mosquito strains, we designed a single gRNA (GAGTGTCCCACGTTAGAAGGAGCGG) that targets coding exon two of the predicted $A G A P 009616$ locus structure (Extended Data Fig. 10b), which codes for amino acids conserved between the majority of Hodorfamily proteins. The gRNA spacer was cloned by Bsal-mediated Golden Gate Assembly using 9616gF (TGCTGTGTCCCACGCTAGAAGGAG) and 9616gR (AAACCTCCTTCTAGCGTGGGACAC) into a U6-expression vector, p125 (available from AddGene), to create p125-9616 containing the U6::gRNA cassette of $165^{63}$, a 3xP3::DsRed marker and piggyBac repeats for germline transformation. To generate transgenic mosquito lines,

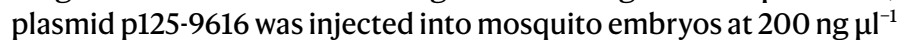

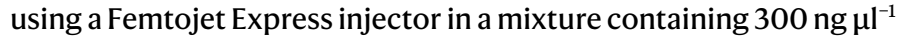
helper vector expressing piggyBac transposase to mediate genomic integration. Surviving $\mathrm{G}_{0}$ individuals were crossed to wild-type mosquitoes, and the progeny was screened under a fluorescent microscope for expression of DsRed to recover $\mathrm{G}_{1}$ transformants. Two independent gRNA-expressing strains were generated by random integration of which one line $\left(g_{10}\right)$ was used in subsequent crosses to generate mutant lines.

\section{Generation, genotyping and phenotyping of A.gambiae hodor} mutant strains

To generate AGAPOO9616 mutant strains, we crossed 10-20 GFPpositive females of vasa:hCas9 line with 10-20 RFP-positive males of 
guide RNA-bearing line $\mathrm{g}_{10}$. We selected 10-20 GFP- and RFP-positive male progeny of this cross, and crossed them en masse to wild-type females. To make sure that no source of Cas9 and gRNA were present in the subsequent generations, we selected GFP- and RFP-negative male progeny of the second cross, and crossed each of these males separately to a batch of 5-10 wild-type females. After collecting the eggs from each single-male cross, males were euthanized and genotyped to determine the presence of a possible mutation in the AGAPOO9616 gene. Among the different mutations we managed to recover three independent mutations that contained 8-bp, 16-bp and 19-bp deletions at the target site. To maintain these three mutant strains, potentially mutant females at each generation were crossed en masse to wild-type males. The pupal progeny was then genotyped by extracting the DNA of pupal exuviae using the QIAGEN DNeasy Blood \& Tissue Kit with a final elution step in $50 \mu \mathrm{l}$ of buffer AE. For each sample, a PCR amplification was set up using the $\mathrm{p} 9616$ forward and reverse primers below, using the following thermocycling conditions: 30 cycles; annealing $67^{\circ} \mathrm{C}$, $30 \mathrm{~s}$; extension $72{ }^{\circ} \mathrm{C}, 30 \mathrm{~s}$. The PCR product was purified (QIAquick PCR Purification Kit, QIAGEN), and around 150 ng of this template was exposed to the restriction enzyme BsrBI (NEB). This restriction enzyme was predicted to cut the wild-type amplicon once, but not the deletion alleles that lacked the restriction site. The purified PCR product was further analysed with Sanger sequencing of amplicons using the p9616seq forward and reverse primers below. To genotype adults, genomic DNA was extracted from adult mosquitoes using the QIAGEN DNeasy Blood \& Tissue Kit with a final elution step in $50 \mu$ l of buffer AE. For each sample, two PCR amplifications were set up. We either used primers p 9616 forward and reverse using the following thermocycling conditions: 30 cycles; annealing $67^{\circ} \mathrm{C}, 30 \mathrm{~s}$; extension $72{ }^{\circ} \mathrm{C}, 30 \mathrm{~s}$, or primers $\mathrm{p} 9616$ forward and pDEL1 (reverse primer designed to bind the wild-type allele) below using the following thermocycling conditions: 30 cycles; annealing $62^{\circ} \mathrm{C}, 30 \mathrm{~s}$; extension $72^{\circ} \mathrm{C}, 30 \mathrm{~s}$. The PCR product was purified (QIAquick PCR Purification Kit, QIAGEN) and about 150 ng was digested with Scal (Thermo Fisher Scientific). Primers: p9616 F/ R: forward, ACGCATTCATAACCAAGACGA; reverse, CGTTTGTAC CGTTGATGGATTC. P9616seq F/R: forward, GACTTAAATCGGCATAG CACTGTG; reverse, CGTTTGTACCGTTGATGGATTC. pDEL1R: reverse, CCACGCTAGAAGGAGCG.

\section{Viability assay for Anopheles mutant strains}

Potentially heterozygous mosquitoes were separated at the pupal stage and were allowed to emerge as adults singly in cups. Exuviae were collected for each individual pupa and were genotyped as described in the previous section. All verified heterozygous individuals were used to set up a sibling cross in cages of $30 \times 30 \times 30 \mathrm{~cm}$ (BugDorm). Generally, 10 females and 10 males were crossed for each experiment. They were allowed to mate for at least 5 days, then fed with screened human blood provided by the National Health Service (NHS) through Hemotek LTD apparatus. Two days later, an egg bowl containing rearing water (distilled $\mathrm{H}_{2} \mathrm{O}$ supplemented with $0.1 \%$ pure salt) was placed in the cage. One or two days after hatching, larvae were placed in trays containing rearing water, allowed to develop as adults and then euthanized and genotyped. Control crosses with wild-type males and females were set up in parallel. The data collected from the control crosses (number of eggs laid, hatching rate, eclosion rate) were compared to the data obtained from the sibling heterozygous mutant crosses.

\section{Reporting summary}

Further information on research design is available in the Nature Research Reporting Summary linked to this paper.

\section{Data availability}

All raw data are available from the corresponding author on reasonable request.
31. Sudarsan, V., Pasalodos-Sanchez, S., Wan, S., Gampel, A. \& Skaer, H. A genetic hierarchy establishes mitogenic signalling and mitotic competence in the renal tubules of Drosophila. Development 129, 935-944 (2002).

32. Phillips, M. D. \& Thomas, G. H. Brush border spectrin is required for early endosome recycling in Drosophila. J. Cell Sci. 119, 1361-1370 (2006).

33. Kim, E., Goraksha-Hicks, P., Li, L., Neufeld, T. P. \& Guan, K. L. Regulation of TORC1 by Rag GTPases in nutrient response. Nat. Cell Biol. 10, 935-945 (2008).

34. Romero-Pozuelo, J., Demetriades, C., Schroeder, P. \& Teleman, A. A. CycD/Cdk4 and discontinuities in Dpp signaling activate TORC1 in the Drosophila wing disc. Dev. Cell 42 376-387.e5 (2017).

35. Pircs, K. et al. Advantages and limitations of different p62-based assays for estimating autophagic activity in Drosophila. PLoS ONE 7, e44214 (2012).

36. Hegedüs, K. et al. The Ccz1-Mon1-Rab7 module and Rab5 control distinct steps of autophagy. Mol. Biol. Cell 27, 3132-3142 (2016).

37. Marois, E., Mahmoud, A. \& Eaton, S. The endocytic pathway and formation of the Wingless morphogen gradient. Development 133, 307-317 (2006).

38. Sun, Q. et al. Intracellular chloride and scaffold protein Mo25 cooperatively regulate transepithelial ion transport through WNK signaling in the Malpighian tubule. J. Am. Soc. Nephrol. 29, 1449-1461 (2018)

39. Schindelin, J. et al. Fiji: an open-source platform for biological-image analysis. Nat. Methods 9, 676-682 (2012).

40. Layalle, S., Arquier, N. \& Léopold, P. The TOR pathway couples nutrition and developmental timing in Drosophila. Dev. Cell 15, 568-577 (2008).

41. Bhatt, P. K. \& Neckameyer, W. S. Functional analysis of the larval feeding circuit in Drosophila. J. Vis. Exp. 51062, e51062 (2013).

42. Shen, P. Analysis of feeding behavior of Drosophila larvae on solid food. Cold Spring Harb. Protoc. https://doi.org/10.1101/pdb.prot069328 (2012).

43. Almeida de Carvalho, M. J. \& Mirth, C. K. Food intake and food choice are altered by the developmental transition at critical weight in Drosophila melanogaster. Anim. Behav. 126, 195-208 (2017).

44. Toshima, N. \& Tanimura, T. Taste preference for amino acids is dependent on internal nutritional state in Drosophila melanogaster. J. Exp. Biol. 215, 2827-2832 (2012).

45. Preibisch, S., Saalfeld, S. \& Tomancak, P. Globally optimal stitching of tiled 3D microscopic image acquisitions. Bioinformatics 25, 1463-1465 (2009).

46. Shin, S. C. et al. Drosophila microbiome modulates host developmental and metabolic homeostasis via insulin signaling. Science 334, 670-674 (2011).

47. Axelsson, L. et al. Genome sequence of the naturally plasmid-free Lactobacillus plantarum strain NC8 (CCUG 61730).J. Bacteriol. 194, 2391-2392 (2012).

48. Erkosar, B. et al. Drosophila microbiota modulates host metabolic gene expression via IMD/NF-KB signaling. PLoS ONE 9, e94729 (2014).

49. Rera, M. et al. Modulation of longevity and tissue homeostasis by the Drosophila PGC-1 homolog. Cell Metab. 14, 623-634 (2011).

50. Palanker Musselman, L et al. A high-sugar diet produces obesity and insulin resistance in wild-type Drosophila. Dis. Model. Mech. 4, 842-849 (2011).

51. Baena-Lopez, L. A., Alexandre, C., Mitchell, A., Pasakarnis, L. \& Vincent, J. P. Accelerated homologous recombination and subsequent genome modification in Drosophila. Development 140, 4818-4825 (2013).

52. Bischof, J., Maeda, R. K., Hediger, M., Karch, F. \& Basler, K. An optimized transgenesis system for Drosophila using germ-line-specific $\varphi \mathrm{C} 31$ integrases. Proc. Natl Acad. Sci. USA 104, 3312-3317 (2007).

53. Lin, Y. F. et al. MIB: metal ion-binding site prediction and docking server. J. Chem. Inf. Model. 56, 2287-2291 (2016).

54. Maier, J. A. et al. ff14SB: improving the accuracy of protein side chain and backbone parameters from ff99SB. J. Chem. Theory Comput. 11, 3696-3713 (2015).

55. Miller, B. R., III et al. MMPBSA.py: an efficient program for end-state free energy calculations. J. Chem. Theory Comput. 8, 3314-3321 (2012).

56. Liman, E. R., Tytgat, J. \& Hess, P. Subunit stoichiometry of a mammalian $\mathrm{K}^{+}$channel determined by construction of multimeric cDNAs. Neuron 9, 861-871 (1992).

57. Misof, B. et al. Phylogenomics resolves the timing and pattern of insect evolution. Science 346, 763-767 (2014).

58. Hervas, S., Sanz, E., Casillas, S., Pool, J. E. \& Barbadilla, A. PopFly: the Drosophila population genomics browser. Bioinformatics 33, 2779-2780 (2017).

59. Charif, D. \& Lobry, J. R. in Structural Approaches to Sequence Evolution (eds Bastolla, U. et al.) 207-232 (Springer, 2007).

60. Li, W. H. Unbiased estimation of the rates of synonymous and nonsynonymous substitution. J. Mol. Evol. 36, 96-99 (1993).

61. Pfeifer, B., Wittelsbürger, U., Ramos-Onsins, S. E. \& Lercher, M. J. PopGenome: an efficient Swiss army knife for population genomic analyses in R. Mol. Biol. Evol. 31, 1929-1936 (2014).

62. Wertheim, J. O., Murrell, B., Smith, M. D., Kosakovsky Pond, S. L. \& Scheffler, K. RELAX: detecting relaxed selection in a phylogenetic framework. Mol. Biol. Evol. 32, 820-832 (2015).

63. Hammond, A. et al. A CRISPR-Cas9 gene drive system targeting female reproduction in the malaria mosquito vector Anopheles gambiae. Nat. Biotechnol. 34, 78-83 (2016).

64. Cong, L. et al. Multiplex genome engineering using CRISPR/Cas systems. Science 339, 819-823 (2013).

65. Volohonsky, G. et al. Tools for Anopheles gambiae transgenesis. G3 (Bethesda) 5, 1151-1163 (2015).

66. Kriventseva, E. V. et al. OrthoDB v10: sampling the diversity of animal, plant, fungal, protist, bacterial and viral genomes for evolutionary and functional annotations of orthologs. Nucleic Acids Res. 47, D807-D811 (2019).

67. Robinson, J. T. et al. Integrative genomics viewer. Nat. Biotechnol. 29, 24-26 (2011).

68. Larkin, M. A. et al. Clustal W and Clustal X version 2.0. Bioinformatics 23, 2947-2948 (2007).

69. Robert, X. \& Gouet, P. Deciphering key features in protein structures with the new ENDscript server. Nucleic Acids Res. 42, W32O-W324 (2014). 
Acknowledgements We thank B. Denholm, T. Perry, E. Prince, A. Rodan, E. Rulifson, A. Teleman, A. Vincent and C. Wilson for sharing reagents; M. Ardakani, D. Dormann, C. Ware and C. Whilding for providing imaging advice; M. Brankatschk, G. Juhasz, F. Missirlis, L. Prieto-Godino, H. Gong and all members of the Miguel-Aliaga, Hirabayashi and Cochemé labs for discussions and experimental advice and/or assistance; E. Knust and W. Huttner for supporting the EM work; and T. Ameku, S. Hirabayashi and S. Vernia for providing comments on an earlier version of this manuscript. S.R. thanks A. M. L. Coenen-Stass, I. Al-Khatib and S. Redhai for advice and support. We thank the Bloomington Drosophila Stock Center, Vienna Drosophila Resource Center, the Kyoto Drosophila Genomics and Genetics Resource for flies, and the Developmental Studies Hybridoma Bank for antibodies. This work was funded by an ERC Advanced Grant to I.M.-A. (ERCAdG 787470 'IntraGutSex'), NIH funding to N.W.B. (ROODK115879), an ERC Starting Grant to N.W. (ERCStG 335724 'VecSyn'), an Imperial Confidence in Concepts grant to I.M.-A., N.W. and S.R., MRC intramural funding to I.M.-A., a BBSRC grant to R.A.B. (BB/LO27690/1), SNF funding to L.v.G. (P40OPB-180894) and an Equipe FRM label to F.L.

Author contributions S.R., C.P. and P.G. performed most Drosophila experiments. L.v.G. and W.-H.L. conducted Xenopus electrophysiology experiments. O.R., F.D., N.D. and P.C.

conducted the Anopheles experiments, T.L. conducted some of the Drosophila developmental and dietary experiments, T.G. conducted the microbiota experiments,
A.M. carried out the western-blot analyses, B.C. (together with C.P.) conducted the genetic screen that led to the identification of hodor, J.B.S. conducted the structural and zincbinding Hodor analyses, Y.-F.W. provided biostatistical/computational expertise, M.Y. and M.W.-B. trained and assisted S.R. with the electron microscopy experiments, M.K.N.L. and N.W. provided advice on the Anopheles experiments, T.W. conducted the phylogenetic analyses, R.A.B. and N.W.B. provided advice on the electrophysiology experiments, and F.L. provided advice on the microbiota experiments. S.R. and I.M.-A. analysed most of the data. I.M.-A. provided conceptual and experimental advice on most experiments and wrote the paper, with contributions from S.R. and inputs from other authors. Most experiments were conducted and analysed by more than one person.

Competing interests The authors declare no competing interests.

\section{Additional information}

Supplementary information is available for this paper at https://doi.org/10.1038/s41586-0202111-5.

Correspondence and requests for materials should be addressed to I.M.-A.

Reprints and permissions information is available at http://www.nature.com/reprints. 


\section{Article}

\begin{tabular}{|c|}
\hline \multicolumn{2}{|c|}{ Screen design } \\
\hline in silico candidate selection \\
\# lines screened
\end{tabular} Vitamin

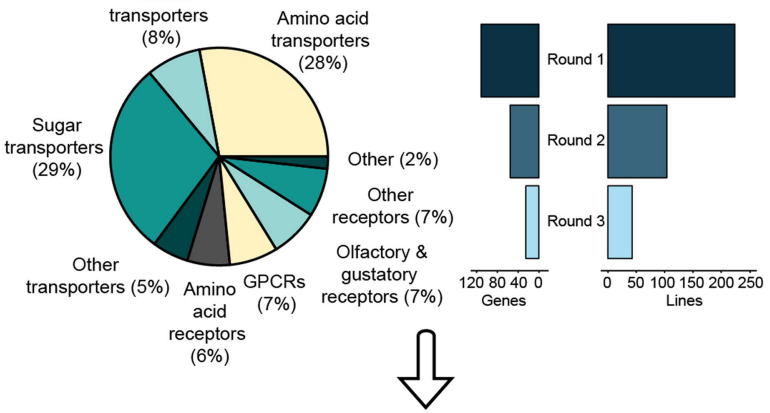

b
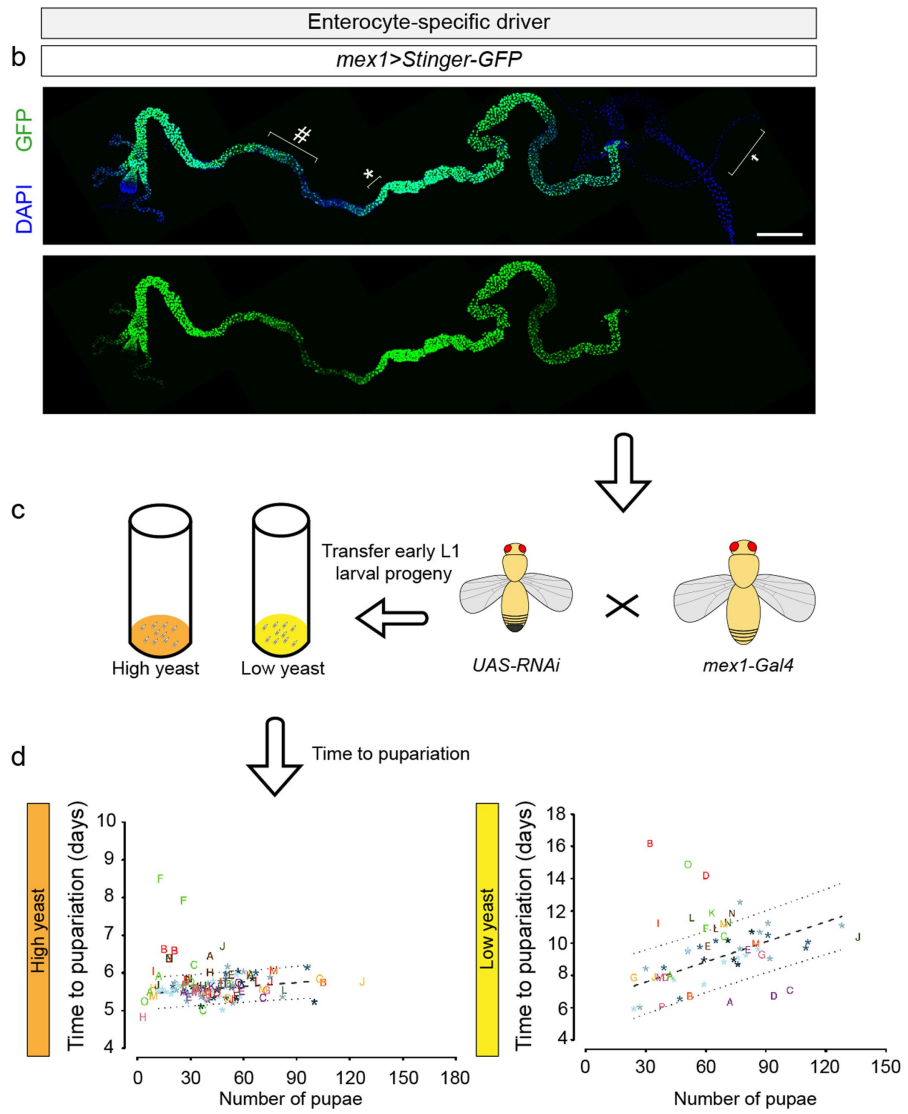

f
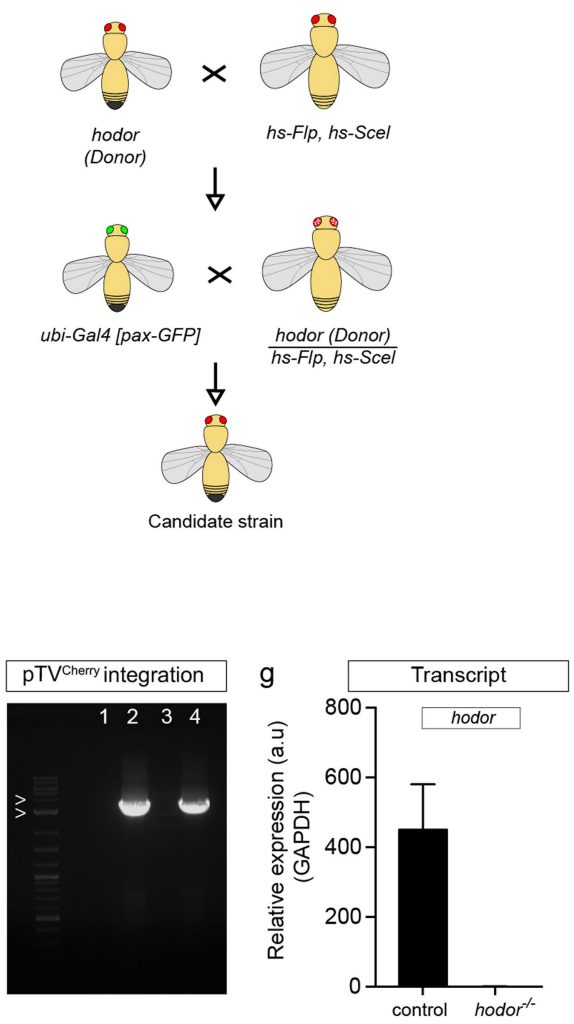

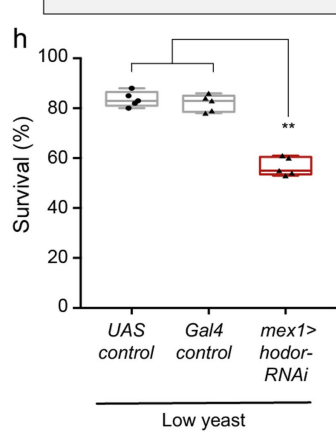

hodor knockdown phenotyping
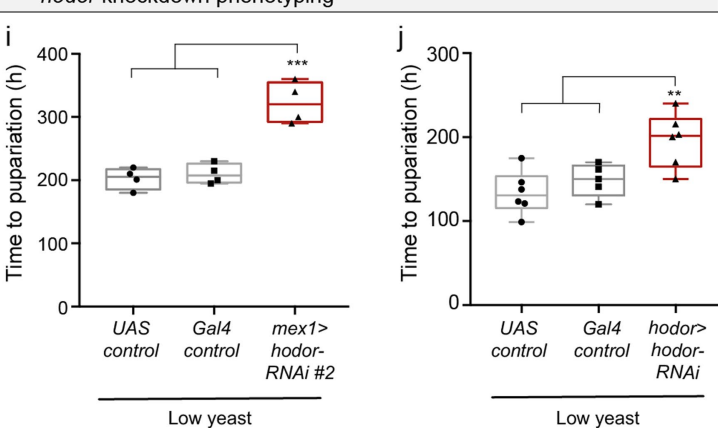

Extended Data Fig. 1 |See next page for caption. 
Extended Data Fig. 1 | Enterocyte screen, hodor mutant validation and hodor knockdown phenotypes. a, Design of enterocyte-specific RNAi-screen and generation of hodor mutant. Distribution of the categories of genes targeted for intestinal knockdown and number of genes and lines tested in each round of the genetic screen. $\mathbf{b}$, Larval gut expressing UAS-Stinger-GFP under the control of mex1-Gal4, showing expression in all enterocytes, including those in the copper cell region (\#) and the iron cell region $(*)$. There is no expression in the Malpighian tubules ( $\dagger$ ). c, Flies carrying UAS-RNAi targeted against candidate genes were crossed to those carrying mex1-Gal4 to achieve enterocyte-specific knockdown in the resulting larval progeny, which were placed on either high- or low-yeast food and allowed to develop into pupae. d, Results from the first round of the RNAi screen using mex1-Gal4 with plots showing the average time to pupariation after egg laying. Blue stars represent four different control lines crossed to mex1-Gal4. Linear models for these control lines (analysed together) are displayed as dashed lines with a 90\% prediction interval shown in dotted lines; knockdown of genes B (CG1134O) and F (CG4797) frequently led to a delay to pupariation. See Source Data for the lines and genes that the specific letters correspond to, and Supplementary
Information for details of-and reasons for-the percentage deviation data display. e, Strategy for generating hodor mutants using $\mathrm{pTV}^{\text {cherry }}$ vector $^{51}$ to direct homologous recombination. Candidate recombinants were recovered after several crosses, identified on the basis of viability and eye colour.f, PCR verification of integration of pTV ${ }^{\text {cherry }}$ construct at the hodor locus, no band is seen in $w^{1118}$ controls $(1,3)$, but a correctly sized band of $3-4 \mathrm{kbp}$ (arrowheads) is seen in hodor $^{+-}(2,4)$. g, Real-time quantitative PCR of control and hodor mutant larvae relative to gapdh, showing the absence of hodor transcripts in the mutant. h, Larval survival in low-yeast conditions when hodor is knocked down in all enterocytes using mex1-Gal4.i, RNAi targeting a different segment of the hodor transcript also causes a developmental delay when expressed with mex1Gal4.j, Limiting expression of hodor RNAi to interstitial cells and principal cells of the Malpighian tubules (using hodor-Gal4) causes a significant delay to development. See Supplementary Information for sample sizes and full genotypes. Scale bar, $1 \mathrm{~mm}$ (b). For cases in which more than two groups were compared, an ordinary one-way ANOVA test was performed with a Tukey post hoc test. ${ }^{*} P<0.05,{ }^{* *} P<0.01,{ }^{* * *} P<0.001$. Box plots: line, median; box, 75 th25th percentiles; whiskers, minimum to maximum. 


\section{Article}

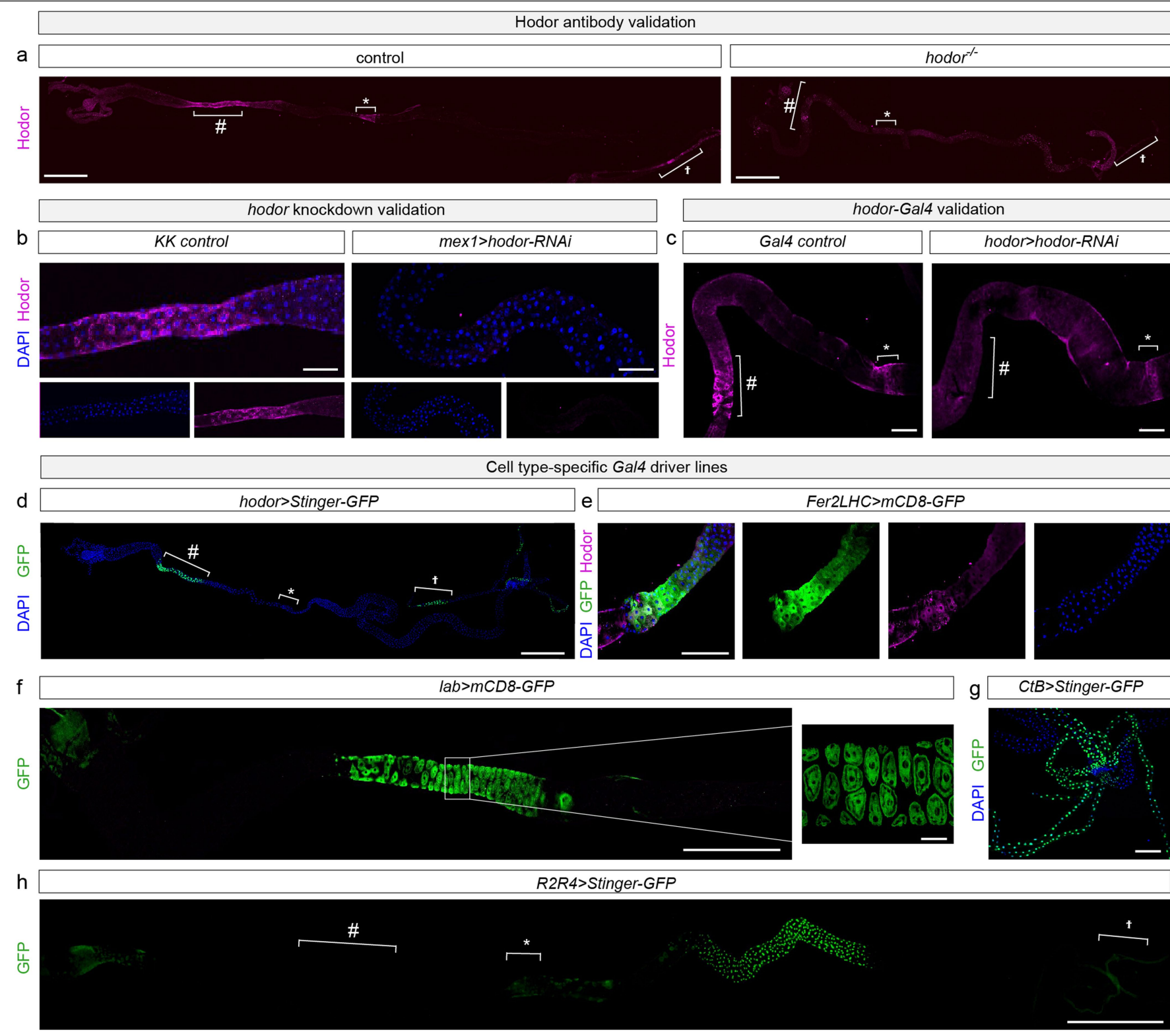

Extended Data Fig. $2 \mid$ Gal4 driver lines used in this study. a, Larval guts stained with anti-Hodor show immunoreactivity in the copper cell (\#) and iron cell $\left({ }^{*}\right)$ regions of the gut and the Malpighian tubules ( $\dagger$ ) in control animals (left), whereas this staining pattern is absent in hodor mutants (right). b, RNAimediated hodor knockdown in enterocytes (using mex1-Gal4) substantially reduces Hodor protein levels. c, RNAi-mediated hodor knockdown using hodorGal4 reduces protein levels considerably in the copper cell region (\#) but does not noticeably reduce levels in the iron cell region $\left({ }^{*}\right)$. d, Expression of GFP in UAS-Stinger-GFPlarvae in interstitial cells (\#) and Malpighian tubules ( $\dagger$ ) using hodor-Gal4; note the absence of GFP in the iron cell region $\left({ }^{*}\right)$.e, Staining of iron cells highlighted in green (Fer2LCH $>m C D 8-G F P$ ) with Hodor antibody illustrating overlap between the two in the anterior portion.f, Expression of $l a b$-Gal4 (visualized in la $b>m C D 8$-GFPlarvae) is seen in the copper cells-but not the interstitial cells-of the copper cell region. The panel to the right shows a higher-magnification image of the copper cell region. $g$, Expression of $C t B$ Gal4 (visualized in CtB $>$ Stinger-GFPlarvae) is confined to the principal cells of Malpighian tubules. h, $R 2 R 4$-Gal4 (visualized in $R 2 R 4>$ Stinger-GFPlarvae) is confined to a subset of enterocytes in the posterior midgut. Note its absence from the copper cell region (\#) and the iron cell region $\left(^{*}\right)$ as well as from Malpighian tubules ( $\dagger$ ). See Supplementary Information for sample sizes and full genotypes. Scale bars, $1 \mathrm{~mm}(\mathbf{a}, \mathbf{d}, \mathbf{f}, \mathbf{h}) ; 200 \mu \mathrm{m}(\mathbf{b}, \mathbf{e}) ; 300 \mu \mathrm{m}(\mathbf{c})$; $200 \mu \mathrm{m}(\mathrm{g}) ; 50 \mu \mathrm{m}$ (f inset). 

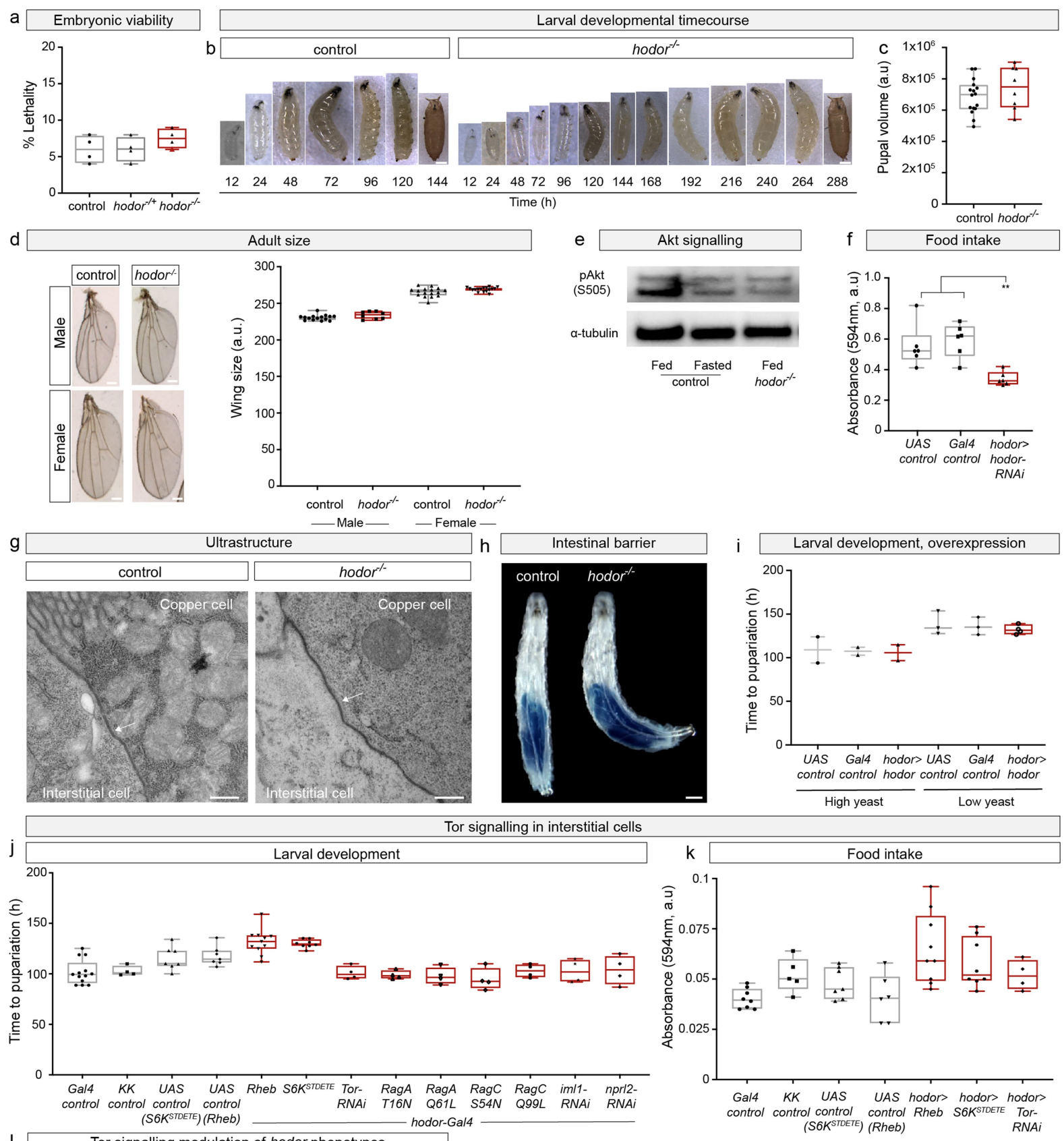

1

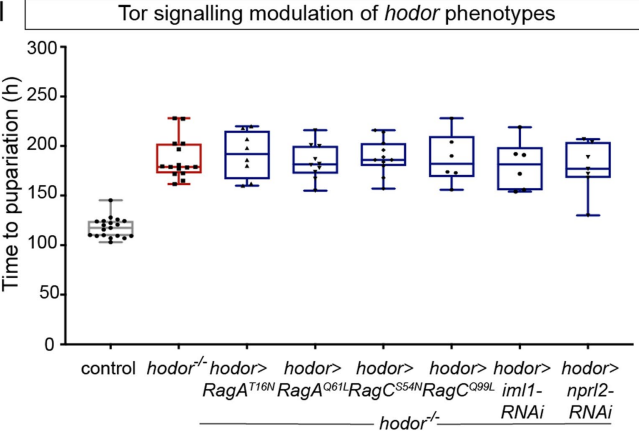

Extended Data Fig. 3 | See next page for caption. 


\section{Article}

Extended Data Fig. 3 Hodor controls food intake and systemic growth. a, Comparison of embryonic viability between control $\left(w^{1118}\right)$, heterozygous $(-/+)$ and homozygous (-/-) hodor mutant larvae; there are no significant differences. b, Developmental progression of larvae lacking hodor compared to control animals $\left(w^{1118}\right)$.c, Pupal volume of hodor mutants compared to controls; each data point represents one pupa.d, Wing size measurements in control compared with hodor mutant adults; no significant differences are apparent (see Methods for details of quantification, each data point represents one wing).e, Reduced levels of pAkt relative to total protein in second-instar hodor mutants compared to controls, all raised on a low-yeast diet. pAkt in hodor mutants is comparable to that of wild-type larvae starved for $15 \mathrm{~h}$.

f, Reduced food intake is seen in hodor-Gal4-driven hodor knockdown larvae when compared to control larvae. Experiments were performed using second instar larvae raised on a low-yeast diet. g, Electron micrographs of the junctional region (arrow) between an interstitial cell and a copper cell, showing no obvious defects in first-instar hodor mutants. $\mathbf{h}$, Smurf assay (Methods) on second-instar control larvae and hodor mutants (examples are representative of at least 6 larvae per genotype). No leakage of blue dye from the intestine was seen in either group. $\mathbf{i}$, Overexpression of hodor in interstitial cells using hodor-gal4 does not alter developmental rate in either high-or low-yeast conditions. j, k, Activation or inactivation of Tor signalling in hodor-expressing cells does not affect developmental rate $(\mathbf{j})$ or food intake (k); none of the genetic manipulations is significantly different compared to its respective control.1, Modulation of Rag and Gatorl complex components in the interstitial cells of hodor mutants (from hodor-Gal4) neither rescues nor exacerbates their developmental delay. See Supplementary Information for sample sizes and full genotypes. Scale bars, $0.5 \mathrm{~mm}(\mathbf{b}) ; 250 \mu \mathrm{m}(\mathbf{d}) ; 500 \mathrm{~nm}(\mathrm{~g})$; $400 \mu \mathrm{m}$ (h). For cases in which more than two groups were compared, an ordinary one-way ANOVA test was performed with a Tukey post hoc test. ${ }^{*} P<0.05,{ }^{* *} P<0.01,{ }^{* * *} P<0.001$. Box plots: line, median; box, 75th-25th percentiles; whiskers, minimum to maximum. 

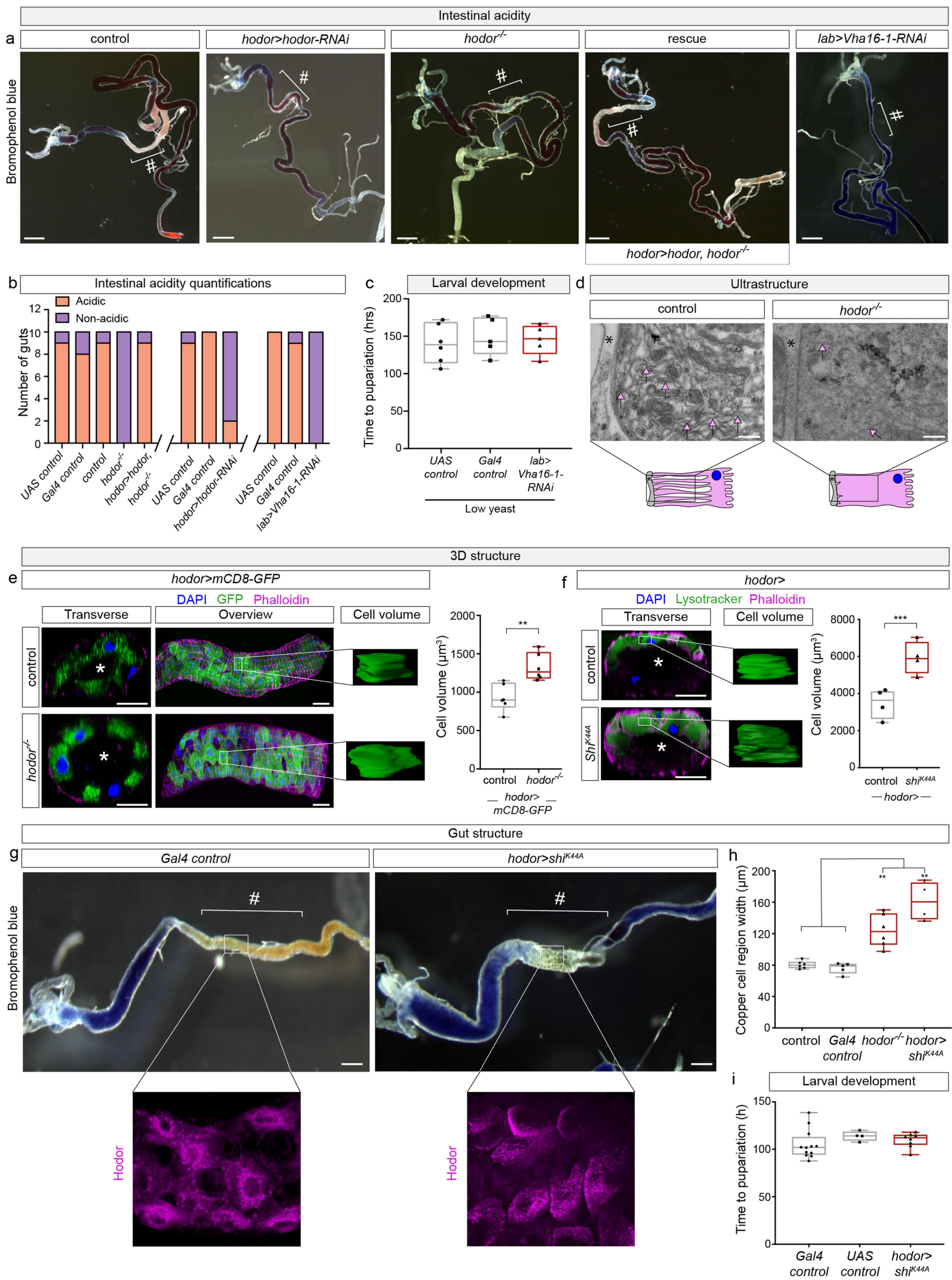

Extended Data Fig. 4 | See next page for caption. 


\section{Article}

Extended Data Fig. $4 \mid$ Hodor sustains luminal acidity and maintains luminal and cell volume. a, The copper cell region (\#) of Drosophila larvae is normally acidic (bromophenol blue dye appears yellow/orange) (Methods), but becomes less acidic (purple/blue) upon expression of hodor RNAi in interstitial cells (hodor-gal4) or in hodor mutants. The reduced acidity of hodor mutant midguts can be rescued by re-expressing hodor in hodor-Gal4-expressing cells. Intestinal acidity is also lost by downregulating the gene that encodes the Vha16-1 subunit of the V-ATPase proton pump in copper cells using lab-Gal4. b, Quantification of intestinal acidity. Depletion (by RNAi) or loss of hodor results in a reduction in the number of larvae with acidic middle midguts, as does depletion of the V-ATPase subunit Vha16-1 in copper cells using lab-gal4. c, Larval developmental rate is unaffected when acidity is lost by reducing the activity of V-ATPase within copper cells (using lab-Gal4).d, Electron micrographs of interstitial cells of first-instar larvae, showing a reduction in their characteristic basal infoldings (arrows) in hodor mutants ( ${ }^{*}$ denotes basa lamina) relative to control cells. e, hodor-Gal4 driven expression of $m C D 8$ - $G F P$ in interstitial cells of control and hodor mutant larvae reveals an increase in luminal volume $\left(^{*}\right)$ and interstitial cell volume (insets with quantifications to the right) in first-instar mutant larvae when compared to controls (all raised on a low-yeast diet). See Methods for details of volume quantifications. f, Overexpression of the dominant-negative Shibire Shi ${ }^{K 4 A}$ in hodor-expressing cells (using hodor-Gal4) reveals an increase in interstitial cell volume in hodor second-instar mutant larvae relative to controls (all raised on a low-yeast diet). LysoTracker staining in green was used to reveal the cytoplasm. Quantifications are shown to the right. Second-instar larvae raised on a low-yeast diet were used for all experiments involving $S h i^{K 44 A}$ expression. $g$, This genetic manipulation also results in an increase in the width of the copper cell region (\#) but does not affect the subcellular localization of Hodor in interstitial cells (insets).h, Quantification of the copper cell region width in controls, hodor mutant larvae and larvae expressing $S h i^{K 44 A}$ from hodor-Gal4. i, Expression of $\mathrm{Shi}^{\mathrm{K44A}}$ in hodor-expressing cells (hodor $>S h i^{K 44 A}$ ) does not alter developmental rate. See Supplementary Information for sample sizes and full genotypes. Scale bars, $500 \mu \mathrm{m}(\mathbf{a}) ; 500 \mathrm{~nm}(\mathbf{d}) ; 10 \mu \mathrm{m}(\mathbf{e}, \mathbf{f}) ; 250 \mu \mathrm{m}(\mathbf{g})$. For comparisons involving two groups, a non-parametric Mann-Whitney $U$-test was used. For cases in which more than two groups were compared, an ordinary one-way ANOVA test was performed with a Tukey post hoc test. ${ }^{*} P<0.05,{ }^{* *} P<0.01$, ${ }^{* * *} P<0.001$. Box plots: line, median; box, 75th-25th percentiles; whiskers, minimum to maximum. 

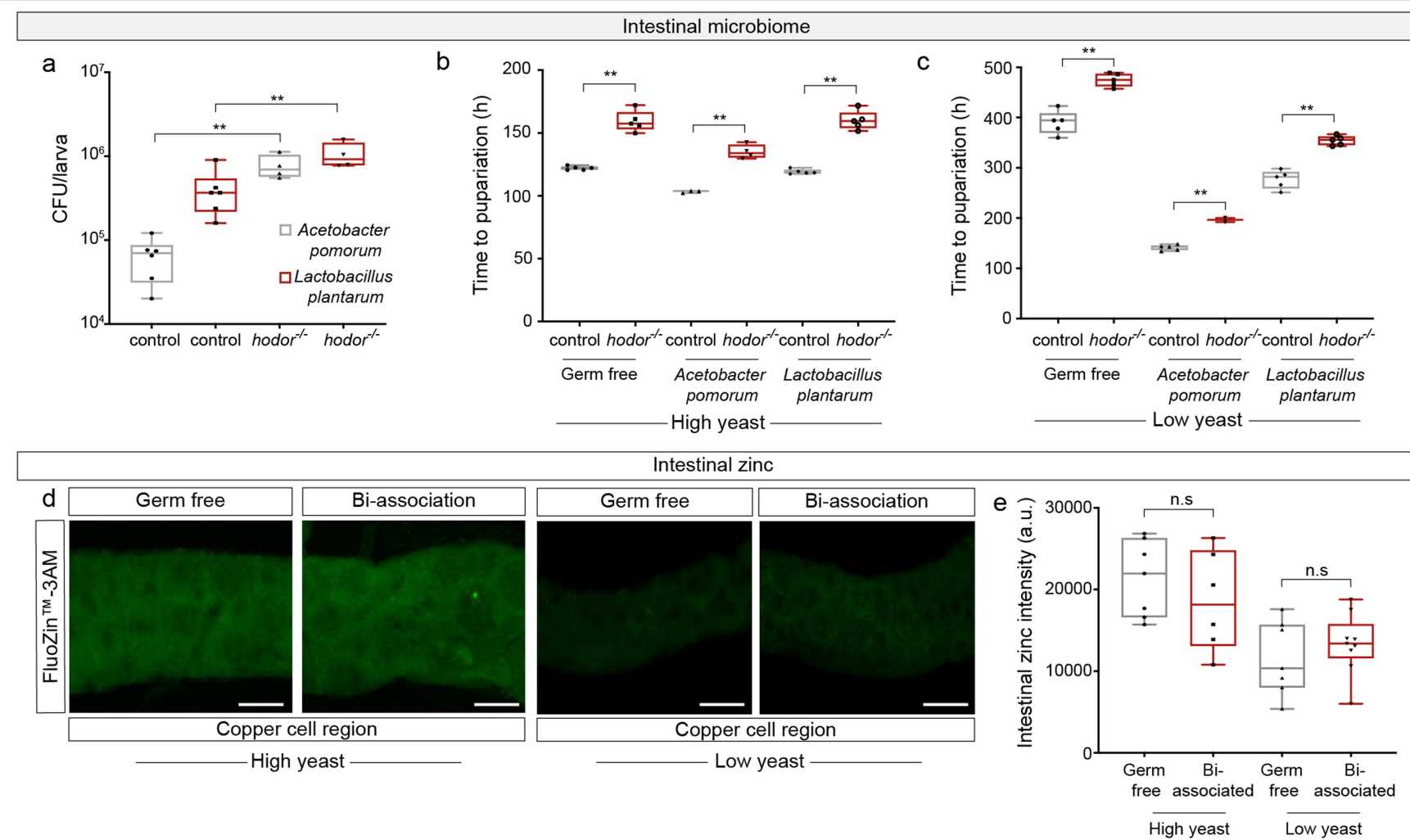

Extended Data Fig. 5 | The microbiota of hodor mutants. a, Increased bacterial loads (cfu per larva) in hodor mutants when compared to control larvae. Bacterial loads were assessed in third-instar larvae raised on a highyeast diet. b, c, Developmental rate of control and hodor mutant larvae in germfree conditions, or following re-colonization with A.pomorum or L. plantarum in either high-(b) or low-yeast (c) conditions. hodor mutants remain developmentally delayed in germ-free conditions, particularly when reared on a low-yeast diet. Mono-association partially rescues the developmental delay of all larvae in low-yeast conditions, but the difference in developmental rate between control and hodor mutant larvae persists. $\mathbf{d}$, Representative images of FluoZin-3AM (a zinc dye) stainings in the copper cell region of larvae reared in germ-free conditions or bi-associated with A. pomorum and $L$. plantarum. More zinc is apparent in the copper cell region of high-yeast-fed larvae relative to low-yeast-fed larvae, but this is unaffected by the presence of microbiota. e, Quantification of zinc staining in the copper cell region. See Supplementary Information for sample sizes and full genotypes. Scale bar, $30 \mu \mathrm{m}$ (d). For comparisons involving two groups, a non-parametric Mann-Whitney $U$-test was used. For cases in which more than two groups were compared, an ordinary one-way ANOVA test was performed with a Tukey post hoc test. ${ }^{*} P<0.05$, ${ }^{* *} P<0.01,{ }^{* * *} P<0.001$. Box plots: line, median; box, 75 th- 25 th percentiles; whiskers, minimum to maximum. 


\section{Article}

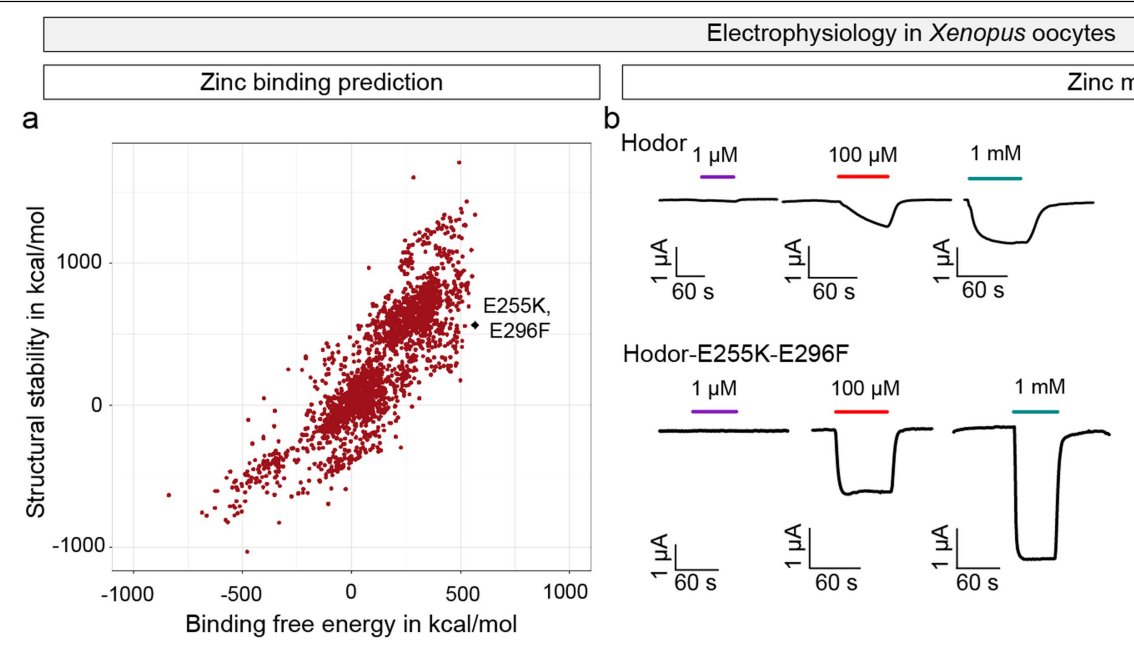

inc modulation of hodor
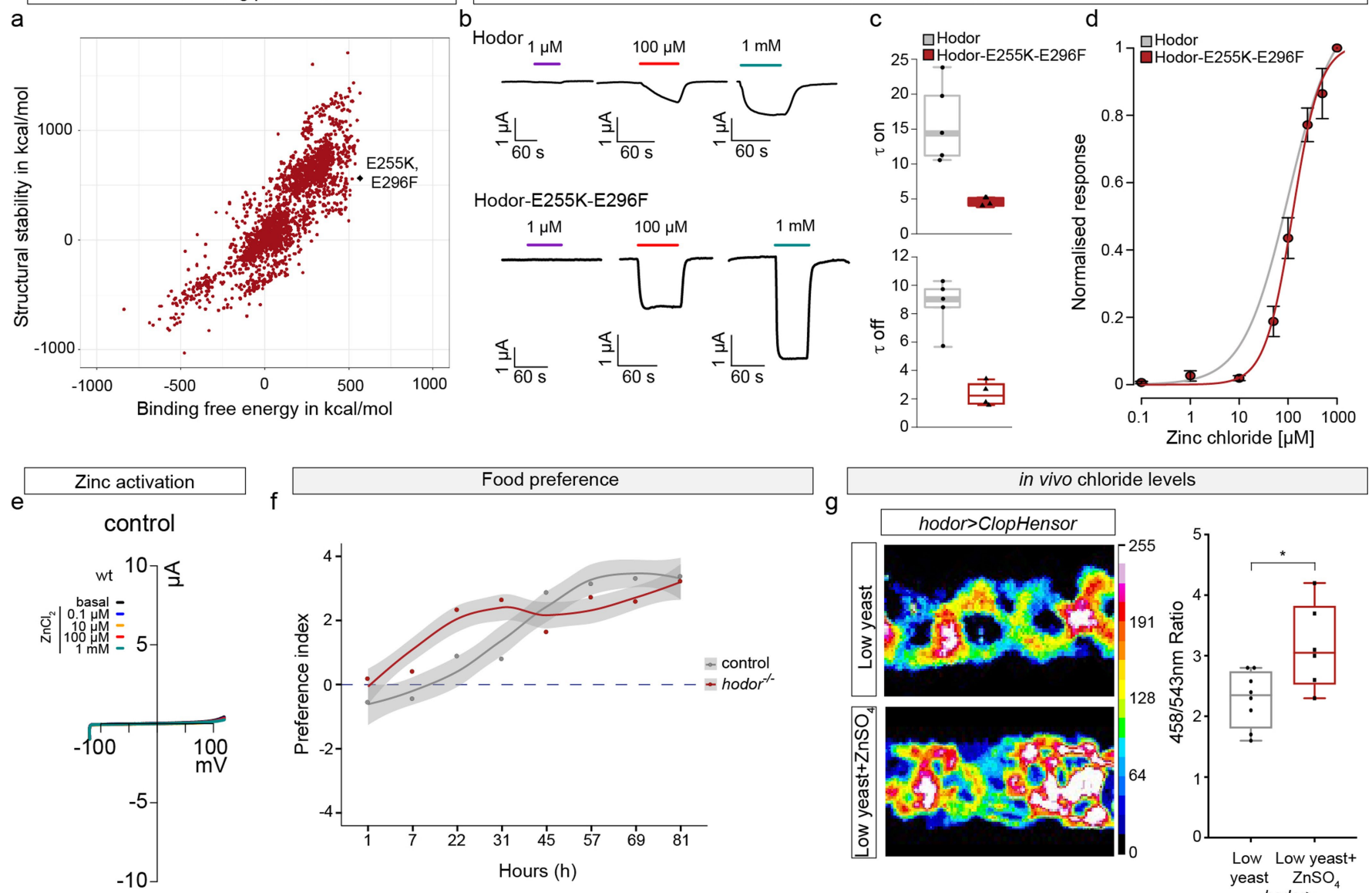

in vivo chloride calibration

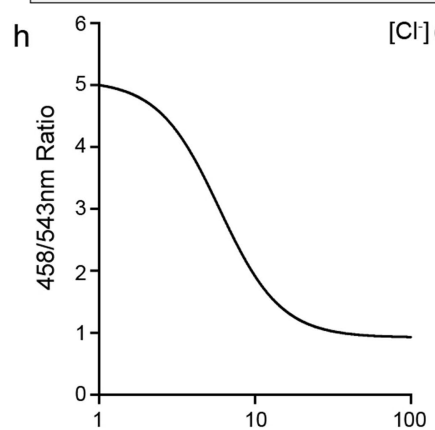

$\left[\mathrm{Cl}^{-}\right](\mathrm{mM})$

Extended Data Fig. 6 | See next page for caption.
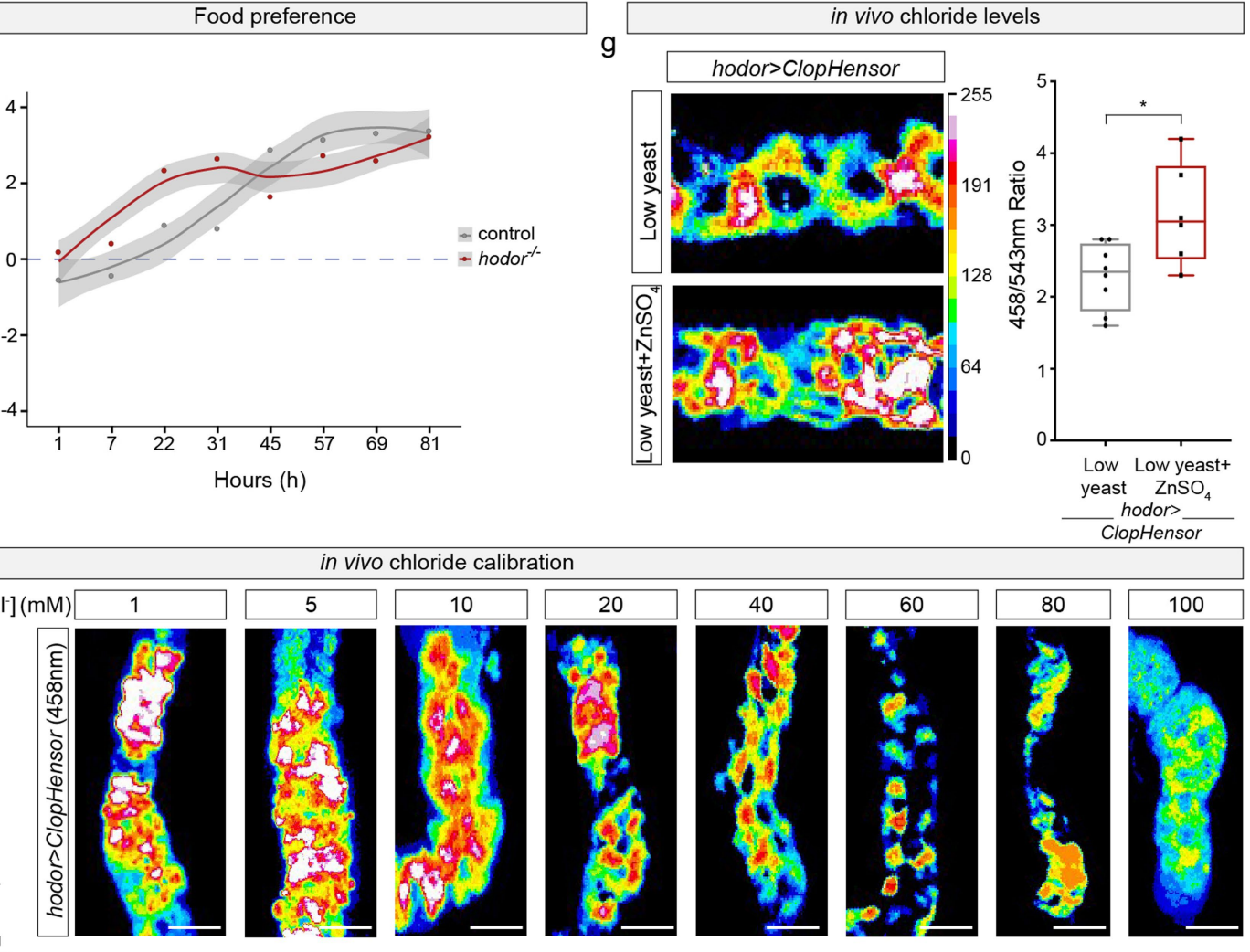
Extended Data Fig. 6 | Hodor gating, transport and effect on food intake.

a, Mutational free energy space, in which structural stability is plotted against zinc binding free energy for each double mutant. The E255K/E296F mutant pair (black dot) was selected to increase the free energy of binding but keep the structural stability as low as possible to avoid refolding of the protein. b, Zincactivated currents from oocytes expressing wild-type Hodor (top) or mutant Hodor(E255K/E296F) (bottom) in response to the indicated concentrations. c, Activation (top) and deactivation (bottom) kinetics of currents elicited by $50 \mu \mathrm{M} \mathrm{ZnCl}_{2}$ were significantly faster in Hodor(E255K/E296F) $(n=4-5, P<0.05$ for ON, $P<0.001$ for OFF, Welsh's $t$-test). $\tau$, time in seconds. d, Concentration dependence of zinc-activated currents from oocytes expressing Hodor (sigmoidal fit from Fig. Bb in grey) compared with that of oocytes expressing Hodor(E255K/E296F) (red). The estimated EC ${ }_{50}$ for Hodor(E255K/E297F) was comparable to that of wild-type Hodor $(119.90 \mu \mathrm{M}, 95 \%$ confidence interval 104.70-137.10 $\mu \mathrm{M})$, with the only significant difference observed in response to $50 \mu \mathrm{MZnCl}_{2}(P<0.05$, two-way ANOVA with post hoc Bonferroni test, $n=5-9)$. Data represented as mean \pm s.e.m., $n$ denotes the number of oocytes. e, Current-voltage $(I-V)$ relationship of zinc-activated currents from uninfected oocytes in response to the indicated concentrations.f, Preference index plotted over time for larvae given a choice between high- and low-yeast diets. Both control and hodor mutant larvae develop a statistically significant preference for a high-yeast diet (positive numbers) after 24 hog, hodor-Gal4driven ClopHensor expression in live interstitial cells reveals a reduction in intracellular chloride levels (increased $458 \mathrm{~nm} / 543 \mathrm{~nm}$ fluorescence emission ratio) in first-instar larvae raised on a low-yeast diet supplemented with $0.4 \mathrm{mM}$ $\mathrm{ZnSO}_{4}$ compared to larvae raised on a low-yeast diet only. Chloride levels decreased from around $8.6 \mathrm{mM}$ in controls to around $5.7 \mathrm{mM}$ in larvae raised on a $\mathrm{ZnSO}_{4}$-supplemented diet, calculated on the basis of the calibration in Extended Data Fig. 6h. Representative fluorescence images $(458 \mathrm{~nm})$ are shown to the left. h, Calibration of the hodor-Gal4 driven fluorescence of ClopHensor in interstitial cells with eight different chloride concentrations (see Methods for details). The calibration graph to the left shows the sigmoidal curve interpolated from individual $458 \mathrm{~nm} / 543 \mathrm{~nm}$ ratios obtained at the different chloride concentrations. This graph enables conversion of absorbance ratios to chloride concentration. Images to the right show representative fluorescence signals (at $458 \mathrm{~nm}$ ) for each concentration. See Supplementary Information for sample sizes and full genotypes. Scale bars, $30 \mu \mathrm{m}(\mathbf{g}, \mathbf{h})$. For comparisons involving two groups, a non-parametric Mann-Whitney $U$-test was used. For cases in which more than two groups were compared, an ordinary one-way ANOVA test was performed with a Tukey post hoc test. ${ }^{*} P<0.05,{ }^{* *} P<0.01,{ }^{* * *} P<0.001$. Box plots: line, median; box, 75th-25th percentiles; whiskers, minimum to maximum. 


\section{Article}

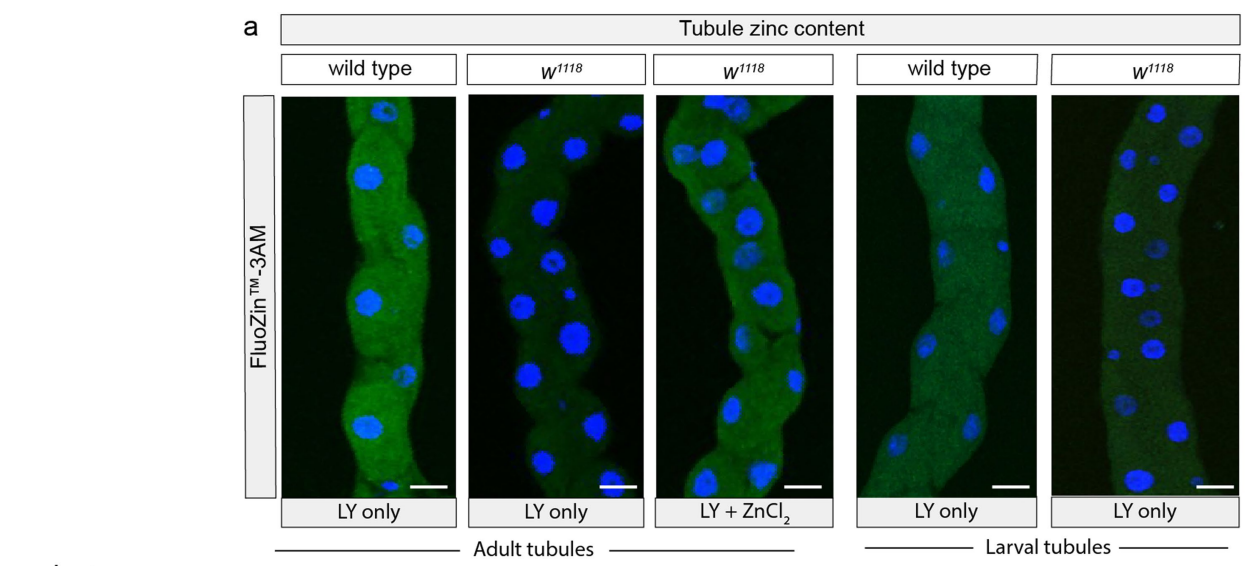

b
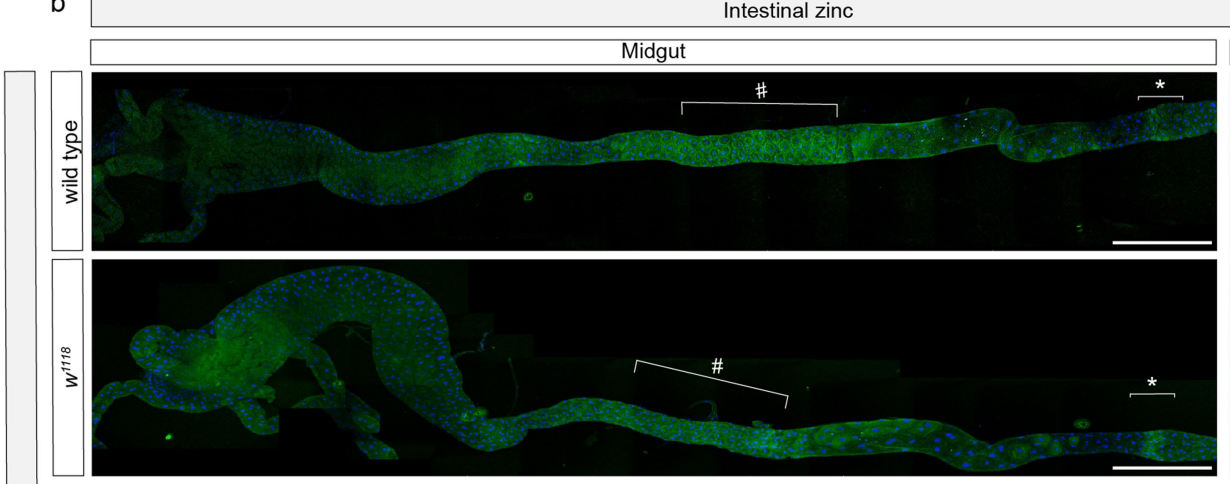

Copper cell region
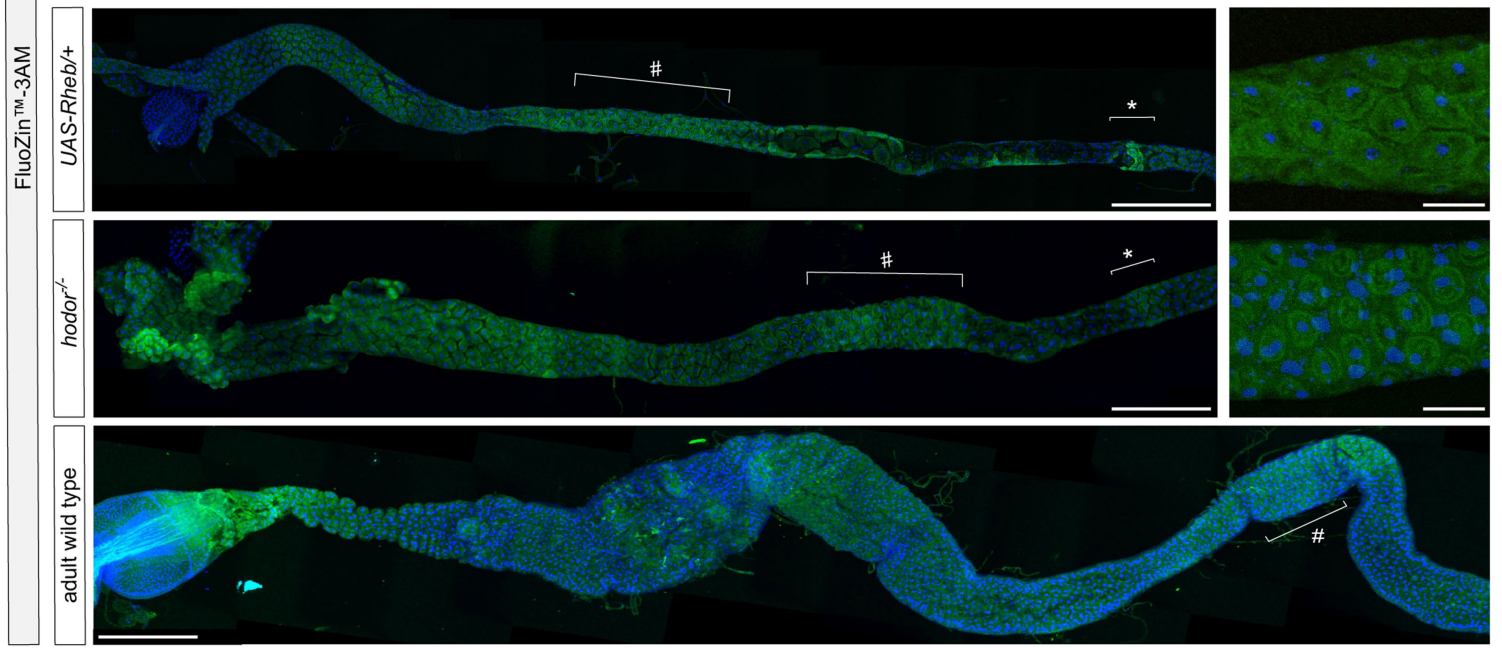

Intestinal zinc quantification
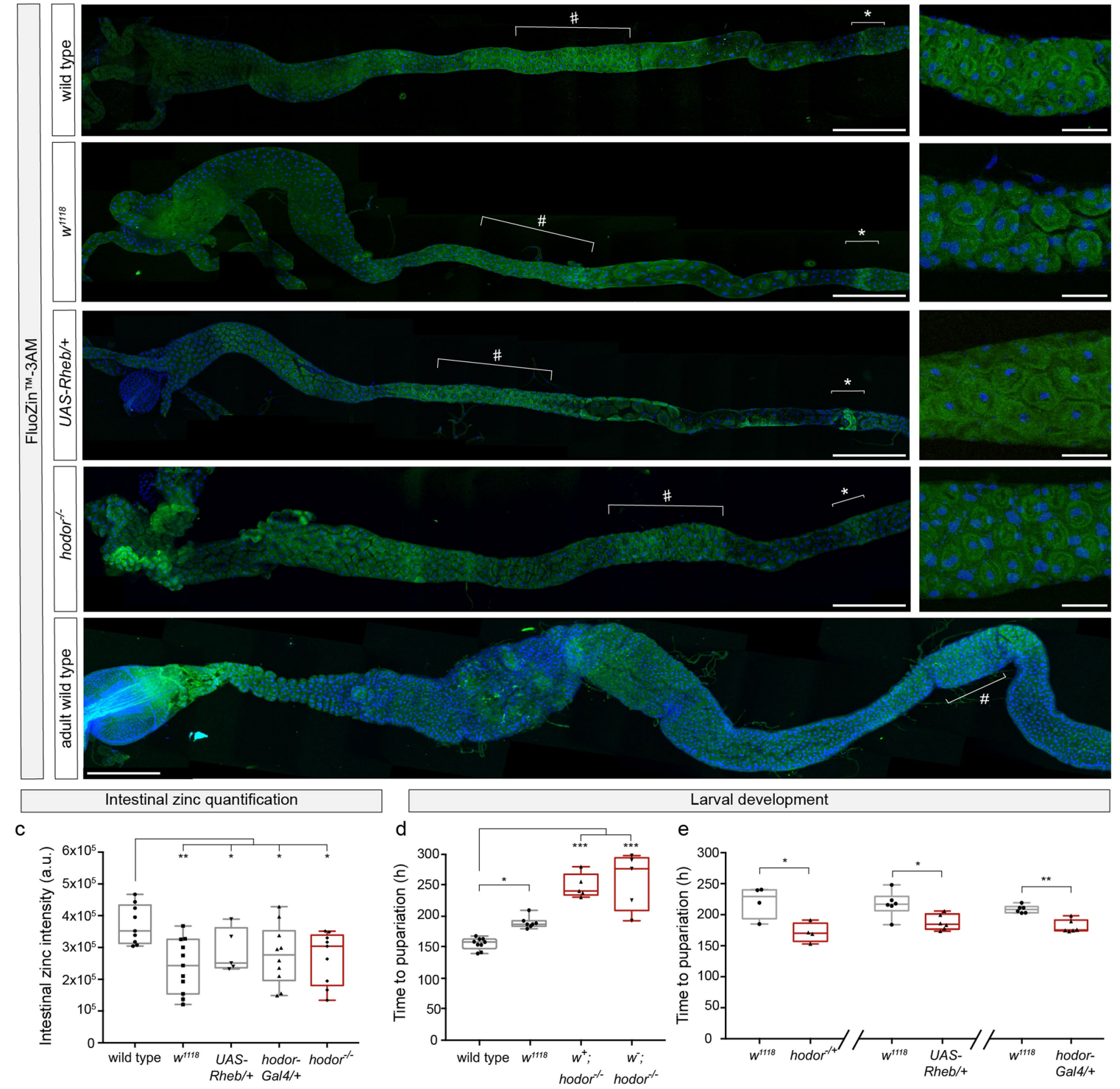

Larval development

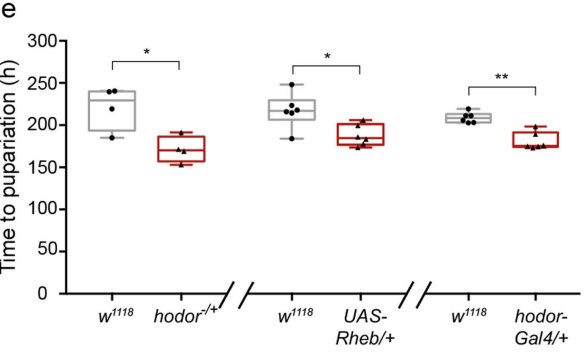

Extended Data Fig. 7 |See next page for caption. 
Extended Data Fig. 7 | Intestinal zinc stainings. a, Validation of the zincsensitive dye, FluoZin-3AM, in adult and larval Malpighian tubules. The tubules of $w^{1118}$ adults have less zinc than those of wild-type (OrR) adults; levels can be increased by supplementing their adult diet with $1 \mathrm{mM} \mathrm{ZnCl}_{2}$ for 3 days (left three panels). A more modest reduction in zinc levels is observed in larval tubules of second-instar $w^{1118}$ larvae relative to wild-type $O r R$ larvae (right two panels). b, FluoZin-3AM staining in the middle midgut of second-instar wildtype larvae (OrR, which harbour a wild-type $w$ gene), $w$ mutant larvae $\left(w^{1118}\right), w$ mutant larvae with a mini- $w$ transgene (UAS-Rheb/+) and hodor mutant larvae (which are mutant for $w$ but carry mini- $w$ transgenes). \# denotes the copper cell region and ${ }^{*}$ denotes the iron cell region. The panels to the right show highermagnification images of the copper cell region. Zinc levels are higher in the copper cell region of wild-type larvae relative to the other genotypes, which have comparable zinc levels. The bottom panel shows FluoZin-3AM staining of a wild-type $(\mathrm{OrR})$ adult midgut. There is no apparent zinc enrichment in the copper cell region (\#).c, Quantification of intestinal zinc in the copper cell region. In both $\mathbf{c}$ and d, larvae were raised on a low-yeast diet. d, Wild-type $O r R$ larvae reach the pupal stage significantly faster than $w^{1118}$ in low-yeast conditions, whereas hodor-/ still causes a significant developmental delay in either a genetic background with an intact $w$ gene $\left(w^{+} ;\right.$hodor $\left.^{-1}\right)$ or when backcrossed eight times into a $w$ mutant background lacking the $w$ gene $\left(w ;\right.$ hodor-- $^{-1}$.e, Heterozygous lines carrying mini- $w$ develop faster than $w^{1118}$ larvae in low-yeast conditions. Scale bars; $50 \mu \mathrm{m}$ (a); $500 \mu \mathrm{m}$ (b); $50 \mu \mathrm{m}$ (inset). See Supplementary Information for sample sizes and full genotypes. For comparisons involving two groups, a non-parametric Mann-Whitney $U$-test was used. For cases in which more than two groups were compared, an ordinary one-way ANOVA was performed with a Tukey post hoc test. ${ }^{*} P<0.05$, ${ }^{* *} P<0.01$, ${ }^{* * *} P<0.001$. Box plots: line, median; box, 75th-25th percentiles; whiskers, minimum to maximum. 


\section{Article}

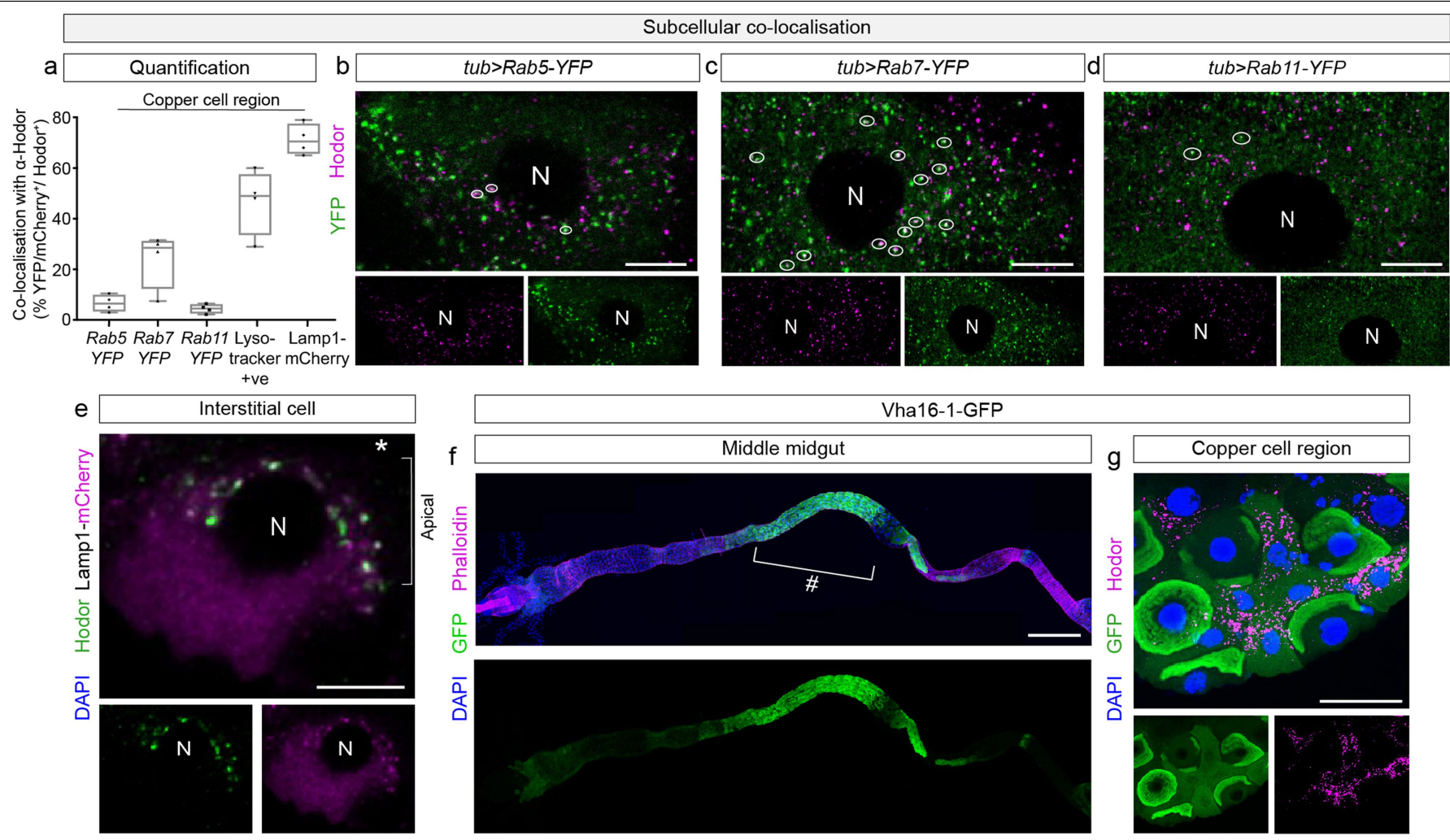

Extended Data Fig. 8 | Subcellular localization of Hodor. a, Quantification of the fraction of Hodor-positive punctae that co-express Rab 5, 7,11 (all of which are endogenously tagged with YFP), Lysotracker or Lamp1 (endogenously expressed Lamp1-mCherry).b-d, Co-expression analysis reveals limited overlap between Hodor immunoreactivity and the early endosome marker Rab5 (b) or the recycling endosome marker Rab11 (d), whereas more pronounced overlap is apparent with late endosome and lysosome marker Rab7 (c).e, The majority of Lamp1-positive structures co-expressed Hodor on the apical side of interstitial cells (* denotes the intestinal lumen). Larvae were briefly starved $(4 \mathrm{~h})$ before dissection in order to visualize lysosomes as punctate structures.f, The endogenously expressed GFP-tagged Vha16-1 subunit of the V-ATPase complex is predominantly localized to the copper cell region (\#) within the larval intestine. g, Expression of Vha16-1-GFP is apparent in both the copper cells and, to a lesser extent, the interstitial cells.

See Supplementary Information for sample sizes and full genotypes. Scale bars, $10 \mu \mathrm{m}(\mathbf{b}-\mathbf{e}) ; 200 \mu \mathrm{m}(\mathbf{f}) ; 30 \mu \mathrm{m}(\mathrm{g})$. N, nucleus. ${ }^{*} P<0.05,{ }^{* *} P<0.01$, ${ }^{* * *} P<0.001$. Box plots: line, median; box, 75 th -25 th percentiles; whiskers, minimum to maximum. 

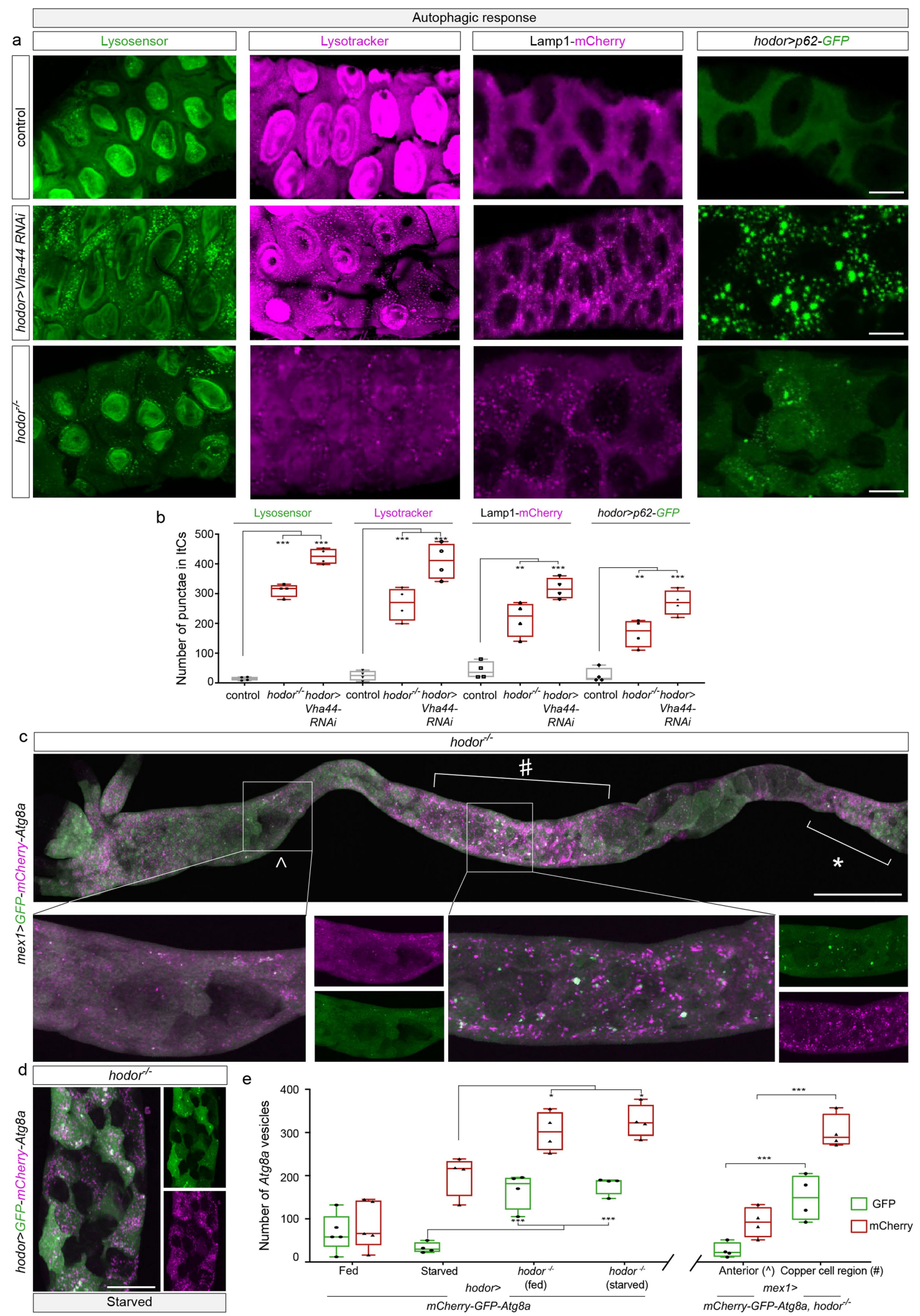

Extended Data Fig. 9 |See next page for caption. 


\section{Article}

Extended Data Fig. 9 |Hodor regulates autophagy. a, Representative expression of LysoSensor, LysoTracker, Lamp1-mCherry and hodor-Gal4driven p62-GFP in the copper cell region of control larvae, larvae in which the V-ATPase subunit Vha44 has been downregulated in interstitial cells (using hodor-Gal4) or hodor mutant larvae. Vha44 knockdown and, to a lesser extent, hodor mutation result in an increase in the number of punctae that are positive for these markers.b, Quantification of the number of punctae that are positive for the abovementioned markers in all three types of larvae shown in a.c, hodo mutants expressing the dual autophagosome/autolysosome marker UAS-GFP. mCherry-Atg8a in all enterocytes (using mex1-Gal4) show regional enrichment of autophagy in both the copper cell region (\#) and the iron cell region $\left({ }^{*}\right)$ when compared to an anterior portion of the gut $\left(^{\wedge}\right)$. Note the appearance of GFPpositive punctae in the copper cell region (\#), which is suggestive of defective autolysosomes unable to quench the GFP signal.d, hodor-Gal4-driven expression of GFP-mCherry-Atg8 $a$ in interstitial cells of starved hodor mutants. Large subcellular compartments positive for both GFP and mCherry are apparent.e, Quantification of GFP-and/or mCherry-positive Atg8a-expressing autophagosomes and autolysosomes in the copper cell region of fed or starved controls, and fed or starved hodor mutants (left graph, Atg8a reporter expressed from hodor-Gal4; right graph, Atg8a reporter expressed from mex1Gal4 in fed hodor mutants). See Supplementary Information for sample sizes and full genotypes. Scale bars, $30 \mu \mathrm{m}(\mathbf{a}) ; 500 \mu \mathrm{m}(\mathbf{c}) ; 45 \mu \mathrm{m}(\mathbf{d})$. For cases in which more than two groups were compared, an ordinary one-way ANOVA test was performed with a Tukey post hoc test. ${ }^{*} P<0.05,{ }^{* *} P<0.01,{ }^{* * *} P<0.001$. Box plots: line, median; box, 75th-25th percentiles; whiskers, minimum to maximum. 
a
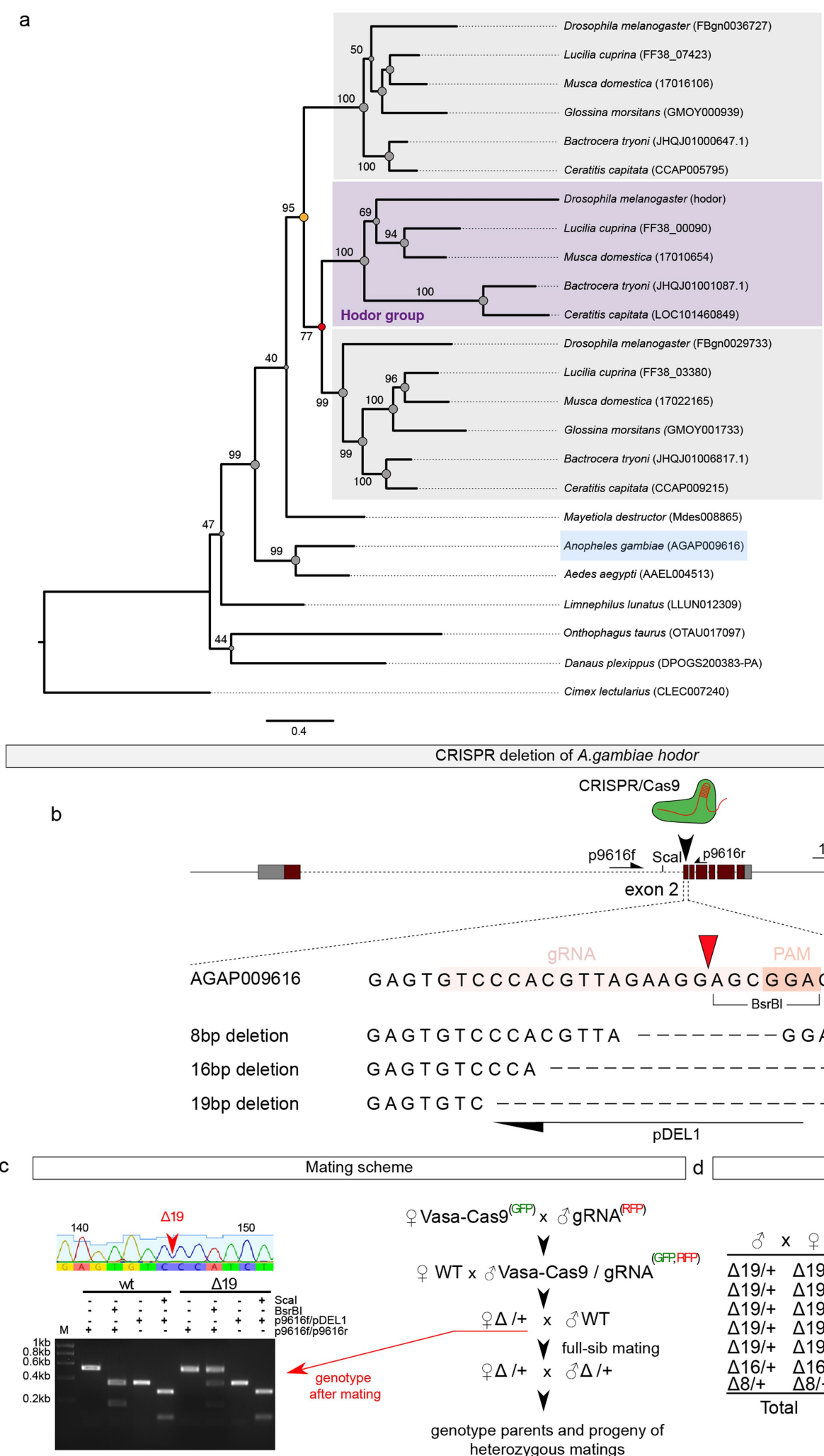

\section{heterozygous matings}

Common fruit fly

Australian sheep blowfly

Housefly

Tsetse fly

Queensland fruit fly

Mediterranean fruit fly

DIPTERA

True flies

| TRICHOPTERA Caddisflies

I COLEOPTERA Beetles

I LEPIDOPTERA Butterflies \& moths

| HEMIPTERA True bugs
Extended Data Fig. $10 \mid$ Hodor is an insect-specific gene, and is essential in A.gambiae.a, Nucleotide-level maximum likelihood phylogeny of the hodor gene family, highlighting successive duplication events at the base of the Schizophora (orange and red nodes, see Methods for details of phylogenetic reconstruction, and Supplementary Information for a complete gene family tree). Bootstrap support is indicated along individual branches as a percentage of 1,000 rapid bootstraps. b, gRNA target site within exon 2 of the $A$.gambiae one-to-many orthologue AGAPOO9616 of fly hodor-like genes, the diagnostic primers used for genotyping and the three frameshift mutants recovered. PAM, protospacer adjacent motif. c, Strategy for the recovery of AGAPOO9616 mutants.d, Genotyping the progeny of crosses between verified heterozygote males and females revealed that AGAPOO9616 homozygous mutant adults are inviable. See Methods for details. 


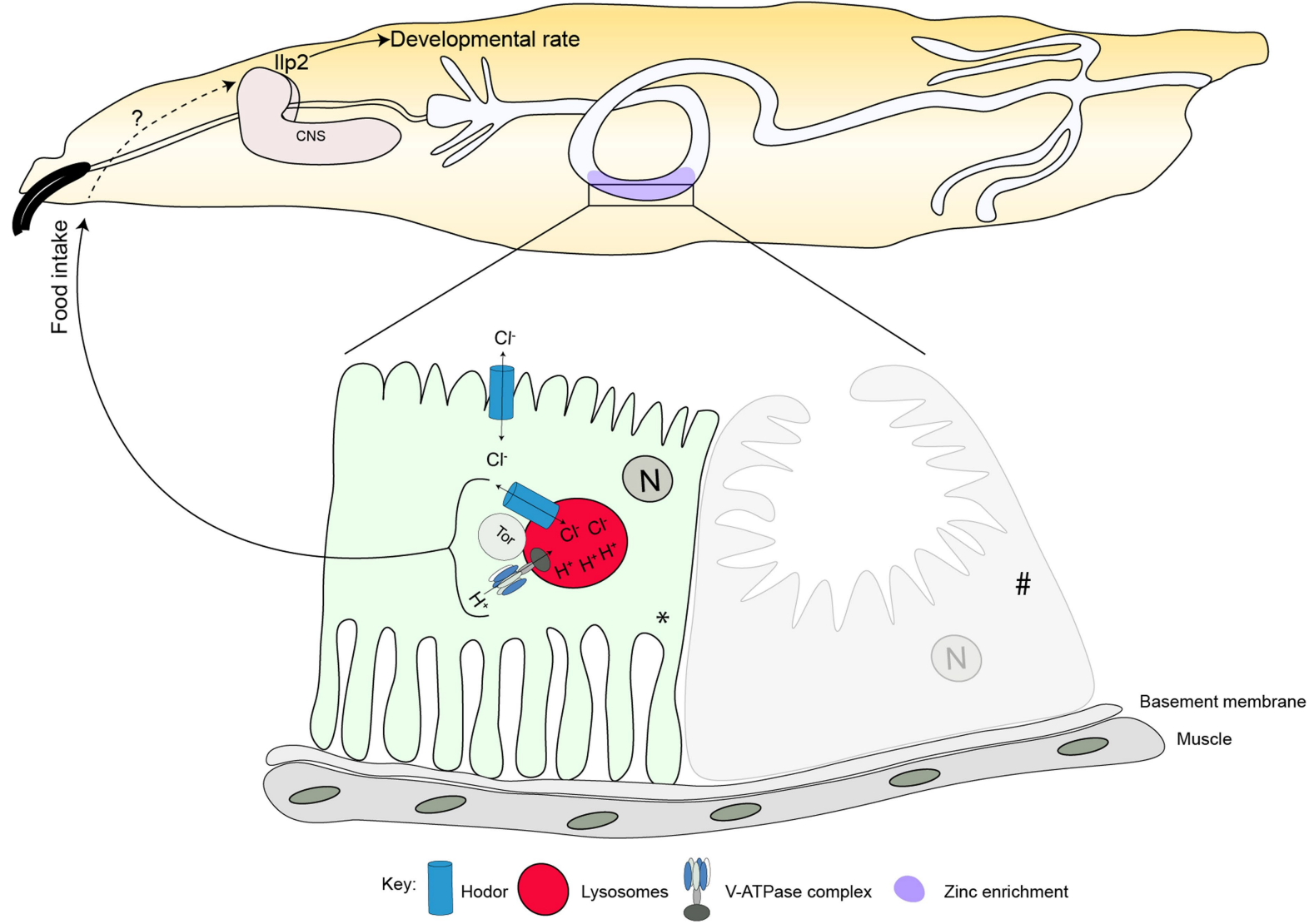

Extended Data Fig. 11 |Current model of Hodor functions. Hodor resides in the apical membrane and on the lysosomes of gut interstitial cells (highlighted in blue, adjacent to acid-secreting copper cells (\#). Zinc sensing by Hodor promotes chloride transport and Tor signalling within interstitial cells. Hodor/ Tor signalling in interstitial cells in turn promotes systemic growth through a neural relay, activating insulin-like signalling and thereby sustaining developmental rate, and by promoting food intake via an as-yet unknown mechanism that is independent of the insulin-producing cells of the brain. The reduced insulin signalling observed in hodor mutants may be secondary to their reduced food intake, hence the dashed arrow. 
Extended Data Table 1 | Compounds tested in Xenopus oocytes

\begin{tabular}{|c|c|c|}
\hline Compound & Concentration & Response \\
\hline Acetylcholine & $10 \mathrm{mM}$ & No response \\
\hline Alanine & $10 \mathrm{mM}$ & No response \\
\hline Arginine & $10 \mathrm{mM}$ & No specific response \\
\hline Asparagine & $10 \mathrm{mM}$ & No response \\
\hline Aspartic acid & $10 \mathrm{mM}$ & No specific response \\
\hline Cysteine & $10 \mathrm{mM}$ & No specific response \\
\hline Cadmium & $500 \mu \mathrm{M}$ & Small response, approximately $233 \pm 70 \mathrm{nA}$ \\
\hline Copper chloride & $500 \mu \mathrm{M}$ & Small response, approximately $20 \mathrm{nA}$ \\
\hline GABA & $10 \mathrm{mM}$ & No response \\
\hline Glutamic acid & $10 \mathrm{mM}$ & No specific response \\
\hline Glutamate & $10 \mathrm{mM}$ & No response \\
\hline Glutamine & $10 \mathrm{mM}$ & No response \\
\hline Glycine & $10 \mathrm{mM}$ & No response \\
\hline Histidine & $10 \mathrm{mM}$ & No specific response \\
\hline Histamine & $10 \mathrm{mM}$ & No response \\
\hline Iron chloride & $500 \mu \mathrm{M}$ & Small response, approximately $20 \mathrm{nA}$ \\
\hline Isoleucine & $10 \mathrm{mM}$ & No response \\
\hline Leucine & $10 \mathrm{mM}$ & No response \\
\hline Lysine & $10 \mathrm{mM}$ & No specific response \\
\hline Methionine & $10 \mathrm{mM}$ & No response \\
\hline Phenylalanine & $10 \mathrm{mM}$ & No response \\
\hline Proline & $10 \mathrm{mM}$ & No response \\
\hline $\mathrm{pH}$ & $\mathrm{pH} 8,9,10$ & Dose-dependent response \\
\hline Serine & $10 \mathrm{mM}$ & No response \\
\hline Serotonin & $10 \mathrm{mM}$ & No specific response \\
\hline Threonine & $10 \mathrm{mM}$ & No response \\
\hline Tryptophan & $10 \mathrm{mM}$ & No response \\
\hline Tyrosine & $10 \mathrm{mM}$ & No response \\
\hline Valine & $10 \mathrm{mM}$ & No response \\
\hline Zinc chloride & $0.1,1,10,50,100,250,500,1000 \mu \mathrm{M}$ & Dose-dependent response, see Fig. $3 b$ \\
\hline Zinc + picrotoxin & $100 \mu \mathrm{M}$ & Did not prevent zinc mediated activation \\
\hline Zinc + pentylenetetrazol & $1 \mathrm{mM}$ & Did not prevent zinc mediated activation \\
\hline
\end{tabular}




\section{Reporting Summary}

Nature Research wishes to improve the reproducibility of the work that we publish. This form provides structure for consistency and transparency in reporting. For further information on Nature Research policies, see Authors \& Referees and the Editorial Policy Checklist.

\section{Statistics}

For all statistical analyses, confirm that the following items are present in the figure legend, table legend, main text, or Methods section.

n/a Confirmed

$\bigotimes$ The exact sample size $(n)$ for each experimental group/condition, given as a discrete number and unit of measurement

$\square$ A statement on whether measurements were taken from distinct samples or whether the same sample was measured repeatedly

$\square$ The statistical test(s) used AND whether they are one- or two-sided

Only common tests should be described solely by name; describe more complex techniques in the Methods section.

Х $\square$ A description of all covariates tested

$\square$ A description of any assumptions or corrections, such as tests of normality and adjustment for multiple comparisons

$\triangle$ A full description of the statistical parameters including central tendency (e.g. means) or other basic estimates (e.g. regression coefficient)

$\triangle$ AND variation (e.g. standard deviation) or associated estimates of uncertainty (e.g. confidence intervals)

$\square$ For null hypothesis testing, the test statistic (e.g. $F, t, r$ ) with confidence intervals, effect sizes, degrees of freedom and $P$ value noted

$\triangle$ Give $P$ values as exact values whenever suitable.

Х $\square$ For Bayesian analysis, information on the choice of priors and Markov chain Monte Carlo settings

Х $\square$ For hierarchical and complex designs, identification of the appropriate level for tests and full reporting of outcomes

$\triangle \square$ Estimates of effect sizes (e.g. Cohen's $d$, Pearson's $r$ ), indicating how they were calculated

Our web collection on statistics for biologists contains articles on many of the points above.

\section{Software and code}

Policy information about availability of computer code

Data collection

Confocal images were acquired Leica SP5 II confocal microscope, a Leica SP5 Inverted microscope and a Leica 10450528 attached to a Leica M165FC stereo microscope using a 0.5x c-mount was used for all other images.

Data analysis

All Image analysis and processing were performed on FIJI version 1.0, Leica LAS AF software and IMARIS 9.2.1. MIB: Metal lon-Binding Site Prediction and Docking software using the fragment transformation method. pClamp 11 and R software was used to analyse and visualise electrophysiology data. Phylogenetic analysis was performed using RaxML v8.1.16 with the following parameters: - $\mathrm{f}$ a -x 12345 p 12345 -\# 1000 -m GTRGAMMA and trees were rendered with FigTree v1.4.2.

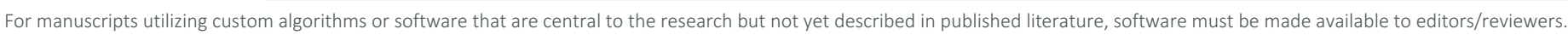
We strongly encourage code deposition in a community repository (e.g. GitHub). See the Nature Research guidelines for submitting code \& software for further information.

Data

Policy information about availability of data

All manuscripts must include a data availability statement. This statement should provide the following information, where applicable:

- Accession codes, unique identifiers, or web links for publicly available datasets

- A list of figures that have associated raw data

- A description of any restrictions on data availability

All data generated or analysed during this study are included in this published article (and its supplementary information files). Further information can be requested from the corresponding author. 
Please select the one below that is the best fit for your research. If you are not sure, read the appropriate sections before making your selection.
$\bigotimes$ Life sciences
Behavioural \& social sciences
Ecological, evolutionary \& environmental sciences

For a reference copy of the document with all sections, see nature.com/documents/nr-reporting-summary-flat.pdf

\section{Life sciences study design}

All studies must disclose on these points even when the disclosure is negative.

Sample size Not limiting for Drosophila experiments. Sample sizes were chosen empirically based on the variability of each scored phenotype. Comparable sample sizes for each genotype/condition were used in every experiment. For sample size information (repeats, \# of animals...) see supplementary table 1.

Data exclusions All experiments were carried out with a positive control which, if it failed to work, made us discard the entire experiment. No data points/ outliers were excluded from our analyses.

Replication All experiments were repeated at least three times yielding comparable outcomes. Further replicates were included if necessary, for example to account for variability resulting from incubator temperature fluctuations or food batch variation.

Randomization Experimental and control flies were bred in identical conditions, and were randomised whenever possible (for example, with regard to housing, position in tray). Control and experimental samples were dissected and processed at the same time and on the same slides.

Blinding

Experimenter was not blind to fly genotypes.

\section{Reporting for specific materials, systems and methods}

We require information from authors about some types of materials, experimental systems and methods used in many studies. Here, indicate whether each material, system or method listed is relevant to your study. If you are not sure if a list item applies to your research, read the appropriate section before selecting a response.

\begin{tabular}{|c|c|c|c|}
\hline \multicolumn{2}{|c|}{ Materials \& experimental systems } & \multicolumn{2}{|c|}{ Methods } \\
\hline $\mathrm{n} / \mathrm{a}$ & Involved in the study & $\mathrm{n} / \mathrm{a}$ & Involved in the study \\
\hline $\mathbf{S}^{-1}$ & $\bigotimes$ Antibodies & Х & $\square$ ChIP-seq \\
\hline$\bigotimes$ & $\square$ Eukaryotic cell lines & Х & $\square$ Flow cytometry \\
\hline$\bigotimes$ & $\square$ Palaeontology & Х & $\square$ MRI-based neuroimaging \\
\hline L & $\bigotimes$ Animals and other organisms & & \\
\hline Х & $\square$ Human research participants & & \\
\hline$\bigotimes$ & $\square$ Clinical data & & \\
\hline
\end{tabular}

\section{Antibodies}

Antibodies used

The following antibodies were used: rabbit anti-Hodor (1:500, this study), mouse anti- $\alpha$-Spectrin (1:10, DSHB \#3A9), anti-Ilp2 (1:200, gift from Pierre Léopold), anti-mCherry (1:200, Thermo Fisher Scientific \#PA534974), p70 S6K (Thr398) (1:1000, Cell signaling \#9209S), pAKT (Ser505) (1:500, Cell Signaling \#4054), tubulin (1:1000, DSHB \#12G10). Conjugated

fluorescent secondary antibodies (FITC-, Cy3- and Cy5) were obtained from Jackson Immunoresearch and used at 1:200. Phalloidin conjugated to AlexaFluor647 or AlexaFluor488 were obtained from Thermo Fisher Scientific (\#A22287) and used at 1:100.

Validation

Hodor antibody was validated in vivo in Drosophila larvae using either knockdown or knockout experiments. For Ilp2, pAKT and p70 S6K antibodies, validation was confirmed with positive controls that would normally induce a change in immunorreactivity (e.g. starvation). For antibodies widely used by the scientific community (e.g tubulin, $\alpha$-Spectrin and mCherry antibodies), validation has either been performed by the manufacturer or previously reported: anti-tubulin - PMID: 11862218, anti- $\alpha$ Spectrin. 
Animals and other organisms

Policy information about studies involving animals; ARRIVE guidelines recommended for reporting animal research

Laboratory animals

Wild animals

Field-collected samples

Ethics oversight
Drosophila melanogaster, A. gambia G3 and Xenopus oocytes including wild type and mutant strains were the only animals used in this study. Stages of Drosophila larvae and Xenopus oocytes are stated in the manuscript.

The study did not involve wild animals.

The study did not involve samples collected from the field.

Experiments involving A. gambiae were approved by the Animal Ethics Committee of Imperial College and are in compliance with United Kingdom Home Office regulations.

Note that full information on the approval of the study protocol must also be provided in the manuscript. 